\title{
REE resource evaluation of some alkaline granites and their weathered crust in South Korea
}

\author{
Shunso Ishihara ${ }^{1}$, Kohei Sato ${ }^{2}$, Yong-Joo Jwa ${ }^{3}$ and Jong-Sun Kim ${ }^{3}$
}

\begin{abstract}
Shunso Ishihara, Kohei Sato, Yong-Joo Jwa and Jong-Sun Kim (2006) REE resource evaluation of some alkaline granites and their weathered crust in South Korea. Bull. Geol. Surv. Japan, vol. 57 (5/ 6), 143-158, 14 figs, 5 tables, 2 appendix tables.
\end{abstract}

\begin{abstract}
A reconnaissance chemical study was made on four granitic bodies in South Korea, on which high REE contents are expected. Triassic foliated granite of the Daegang body occurring along the boundary between the Yeongnam-Ogcheon terrains contains average REE+Y content of $414 \mathrm{ppm}$ and the L/HREE ratio is 8.4. Triassic Hamchang granite of the Ogcheon terrain is composed of highalkali and high-REE group, averaged as $508 \mathrm{ppm}$ REE+Y, with L/HREE=19.0, and low-alkali and low-REE group averaged as $127 \mathrm{ppm} \mathrm{REE}+\mathrm{Y}$ with L/HREE=23. On the contrary, the early Jurassic Hapcheon syenite at southeastern margin of the Yeongnam massif is low in REE+Y (171-217 ppm). The Paleogene Gigye granite in the Gyeongsang basin, which can be a split-off part along the Yangsan fault from the REE-rich alkaline Namsan body, contains 200-300 ppm REE+Y.

Soil samples were collected from the weathered crust of these granitoids and compared with those of unweathered granitoid values. The soil values are generally lower than those of the unweathered granites in the Daegang granite (414 ppm REE+Y in rock vs. 240 ppm REE+Y in soil) and Gigye granite (342 vs. 243 ppm REE+Y), indicating most of the REE leached out during the weathering. The loss-and-gain is unclear on the Hamchang granite and Hapcheon syenite. No clear enrichment of REE during weathering has been so far observed on the granitic bodies in South Korea.
\end{abstract}

Keywords: South Korea, Mesozoic, granitoids, A-type, weathered crust, REE

\section{Introduction}

Rare earth element (REE) mineral resources have become important in the recent so-called high-technology industry. Finding of new REE resources, heavy REE in particular, will be a great necessity in the coming years (Ishihara and Murakami, 2005). Recent supply of REE resources in the world heavily rely on the Bayun Obo mine, Inner Mongolia, China, because of stopping of mining operation at the Mountain Pass carbonatite deposits in California, U.S.A.

Major REE-bearing ore deposits occur associated with carbonatite, alkaline granites, hydrothermal deposits of granitic affinities. There are unique ore deposits of ion-absorption type in southern China (Wu et al., 1990). In these REE deposits, rare earth components are absorbed in the weathered clay minerals in granitic crusts with the enrichment factors up to 2-3 (Ishihara and Murakami, 2005). REE resources of this kind have been rarely studied at outside of the southern China but in Thailand (Kamitani and Hirano, 1994).

In this report, a reconnaissance study was made in
South Korea, visiting four alkaline granitic bodies (Fig.1) where high REE contents have been known sporadically. Samples were taken from fresh granites and nearby weathering crust. Decomposed granite is divided downwards into (i) surface soil with organic matter (A horizon), (ii) completely weathered next horizon (B horizon), and (iii) weakly weathered horizon below it ( $\mathrm{C}$ horizon). The best enrichment of REE is generally obtained from the B horizon (Huang et al., 1989; Wu et al, 1990). Therefore, soil from the B horizon was collected generally, and its REE composition was compared with that of the nearby fresh granites. During this field work, the A horizon was found poorly developed in South Korea.

Localities of the analyzed samples are listed in Table 1 , and the analytical results are given in Tables 2 to 4 .

\section{General Geology}

South Korea is divided into four tectonic provinces with the northeasterly trends; namely, from north to south, Gyeonggi massif, Ogcheon belt, Yeongnam

\footnotetext{
${ }^{1}$ National Institute of Advanced Industrial Science and Technology (AIST), Central 7, 1-1-1 Higashi, Tsukuba 305-8567, Japan.

${ }^{2}$ Institute for Geo-Resources and Environment, GSJ, Central 7, 1-1-1 Higashi, Tsukuba 305-8567, Japan.

${ }^{3}$ Gyeongsang National University, 900 Gajwa-dong, Jinju, 660-701 South Korea.
} 

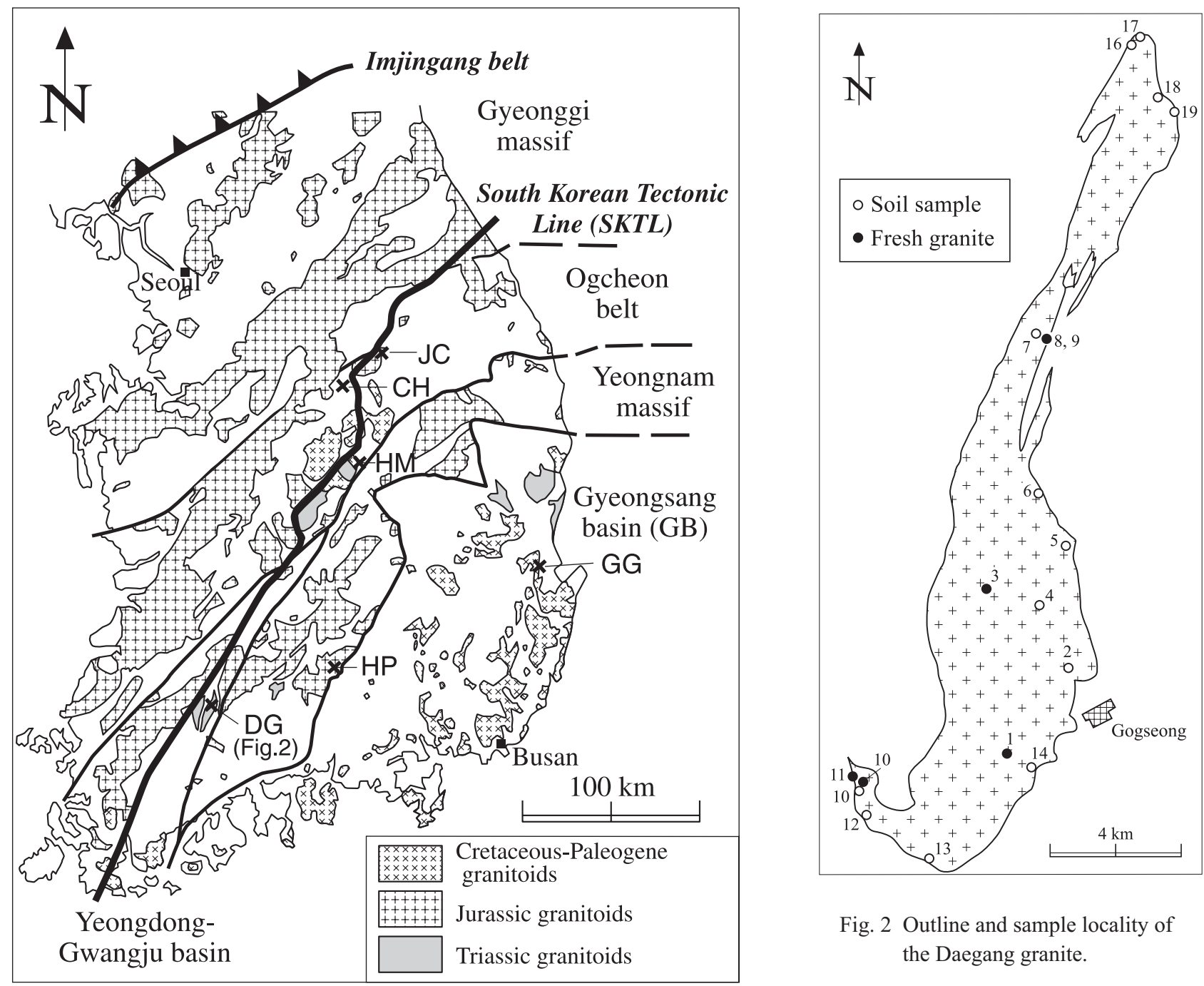

Fig. 2 Outline and sample locality of the Daegang granite.

Fig. 1 Tectonic division and studied areas in South Korea. 1. Daegang granite, 2. Hamchang granite, 3. Gigye granite, 4. Hapcheon syenite. $\mathrm{CH}$, Chungju REE magnetite deposit; JC, Jecheon granite (Ishihara et al., 2005).

massif and Gyeongsang basin (Fig. 1). The Gyeonggi massif and Yeongnam massif are Precambrian metamorphic terrains, but the Ogcheon belt is composed of Paleozoic sedimentary and metamorphic rocks. These terrains are intruded by Mesozoic granitoids. The southeastern corner is Cretaceous to Paleogene sedimentary-volcanic basin intruded by coeval gabbroids and granitoids.

The granitoids are mostly Jurassic and Cretaceous but locally Triassic and Paleogene in age. They are generally composed of Triassic deep-seated ilmenite-series stock, Jurassic deep-seated ilmenite-series batholiths and the Cretaceous-Paleogene magnetiteseries volcano-plutonic complexes (Jwa, 2004; Jin et al., 2001). Almost all the granitoids belong to calcalkaline series, but associated with small alkaline-series bodies, which can be called A type (Koh et al., 1996; Kim et al., 1998).

\subsection{Daegang granite}

The Daegang granite occurs along the tectonic boundary between the Yeongnam massif and the Ogcheon belt, and forms a narrow elongated body in NNE-SSW direction (Fig. 2). The basement rocks are mainly composed of the Precambrian metamorphic complex with intercalation of amphibole schist and crystalline limestone (Kim et al., 1990; Kim and Kim, 1990). Age-unknown metasedimentary rocks cover unconformably the basement. The Daegang granite intruded both the basement and the metasedimentary rocks during Triassic time. Radiometric ages for the granite were reported as $228 \pm 5 \mathrm{Ma}$ by $\mathrm{Rb}-\mathrm{Sr}$ wholerock method (Choo and Kim, 1986), and 212 \pm 2 Ma by $\mathrm{U}-\mathrm{Pb}$ zircon dating (Turek and Kim, 1995).

The Daegang granite is coarse-grained gray granite usually foliated in a north-south direction. The foliation is parallel to the elongation of the plutonic body, 
Table 1 Locality, rock description and magnetic susceptibility of the analyzed fresh samples in South Korea.

\begin{tabular}{|c|c|}
\hline Numbers & Rock types and magnetic susceptibility (MS) \\
\hline \multicolumn{2}{|c|}{ Daegang granite, Gokseong-gun, Jeollanam-do } \\
\hline $\mathrm{R} 1$ & Coarse, biotite granite, foliated; $\mathrm{MS}=6.10 \times 10^{-3} \mathrm{SI}$ \\
\hline R3 & Coarse, biotite granite, foliated; $\mathrm{MS}=1.60 \times 10^{-3} \mathrm{SI}$ \\
\hline R3e & Fine, enclave in the above granite; $\mathrm{MS}=0.16 \times 10^{-3} \mathrm{SI}$ \\
\hline R3a & Medium, dike-like aplite; $\mathrm{MS}=6.12 \times 10^{-3} \mathrm{SI}$ \\
\hline R8 & Coarse, red biotite granite, foliated strongly; $\mathrm{MS}=0.12 \times 10^{-3} \mathrm{SI}$ \\
\hline R9 & $100 \mathrm{~m}$ west of R8. Medium, biotite granite, foliated; $\mathrm{MS}=0.10 \times 10^{-3} \mathrm{SI}$ \\
\hline $\mathrm{R} 10$ & Medium, biotite granite, strongly foliated in parts; $\mathrm{MS}=0.11 \times 10^{-3} \mathrm{SI}$ \\
\hline R11a & Fine, leucogranite, foliated and pegmatitic; MS $=0.06 \times 10^{-3} \mathrm{SI}$ \\
\hline \multicolumn{2}{|c|}{ Hamchang granite, Sangju-si, Gyeongsangbuk-do } \\
\hline $\mathrm{R} 21$ & Anryeonji dam site. Coarse, biotite granite; $\mathrm{MS}=0.14$ \\
\hline R22 & ditto. Fine granodiorite, pink Kf; MS $=0.14 \times 10^{-3} \mathrm{SI}$ \\
\hline $\mathrm{R} 22 \mathrm{~m}$ & ditto. Medium, granodiorite, foliated weakly; $\mathrm{MS}=0.10 \times 10^{-3} \mathrm{SI}$ \\
\hline R28 & ditto. Coarse, biotite granite, pink Kf; MS $=0.12 \times 10^{-3} \mathrm{SI}$ \\
\hline R29 & ditto. Coarse, biotite granite, pink Kf; $\mathrm{MS}=0.18 \times 10^{-3} \mathrm{SI}$ \\
\hline \multicolumn{2}{|c|}{ Gigye granite, Pohang-si, Gyeongsangbuk-do } \\
\hline $\mathrm{R} 30$ & Gigye quarry. Fine biotite granite, micro-pegmatitic; $\mathrm{MS}=0.95 \times 10^{-3} \mathrm{SI}$ \\
\hline R31 & $400 \mathrm{~m}$ south of R30. Fine biotite granite, porphyritic; $\mathrm{MS}=0.26 \times 10^{-3} \mathrm{SI}$ \\
\hline \multicolumn{2}{|c|}{ Hapcheon syenite, Hapcheon-gun, Gyeosangnam-do } \\
\hline R33 & Daepyong. Medium, gray monzonite; $\mathrm{MS}=40.2 \times 10^{-3} \mathrm{SI}$ \\
\hline R34 & $5 \mathrm{~km}$ east of R33. Coarse, biotite syenite; $\mathrm{MS}=17.1 \times 10^{-3} \mathrm{SI}$ \\
\hline R35 & $\begin{array}{l}\text { Daepyong, water fall; fine reddish microgranite sheet }(20 \mathrm{~cm} \text { wide }) \text {; } \\
\mathrm{MS}=2.9 \times 10^{-3} \mathrm{SI}\end{array}$ \\
\hline
\end{tabular}

and magnetite-series granite porphyry. Since the deformed structure observed in the granitoids seems likely to have been caused by Jurassic Kumiri shear deformation (Hwang et al., 1992), the emplacement of the granitoids must have been earlier than the Jurassic shear event.

The Jeomchon granite has U-Pb sphene ages of $224 \pm 3 \mathrm{Ma}$ and $225 \pm 8$ Ma (Sagong et al., 2005), implying it is Triassic in age. The Hamchang granite and associated granite porphyry, however, are considered younger. These rocks are biotite granites in modal composition having the modal feldspar ratio of monzogranite and syenogranite, and in part alkali granite (Choe and Jwa, 1998).

Measurement of magnetic susceptibility indicates low values, below 0.2 $\mathrm{x} 10^{-3} \mathrm{SI}$, which are equivalent to those of the ilmenite series.

\subsection{Gigye granite}

The Gigye granite occurs as a small stock in the middle part of the sedi-

which is strongly sheared locally. The foliation is considered caused by tectonic stress during the intrusion of the original magmas. This granite essentially belongs to the ilmenite-series of Ishihara (1977), because the magnetic susceptibility is generally lower than 0.2 $\mathrm{x} 10^{-3} \mathrm{SI}$ unit (Table 1), except for the R1 of $6.1 \times 10^{-3} \mathrm{SI}$, which exceeds the upper limit of ilmenite series of 3.0 $\mathrm{x} 10^{-3} \mathrm{SI}$.

The Daegang granite is represented by one feldspar hypersolvus granite containing small amounts of mafic silicates, and is classified to an alkali feldspar granite in the IUGS discrimination (Kim et al., 1998). The mafic silicates are small amounts of Fe-rich annite accompanied with alkaline amphibole of riebeckite. The granite has very similar mineralogical and geochemical characteristics to the A-type granites (Kim et al., 1998).

\subsection{Hamchang granite}

The Hamchang granite occurs in the middle part of the Ogcheon belt and forms a stock. The granite intruded the Precambrian metamorphic rocks, age-unknown metasedimentary rocks and the Paleozoic sedimentary rocks, but it was unconformably overlain by the Mesozoic sedimentary rocks (Choi et al., 1993).

Choe and Jwa (1998) considered the Hamchang granite as a member of the Jeomchon granite complex, which is composed of the Paleozoic-Triassic ilmenite-series hornblende-biotite granodiorite and deformed biotite granite, and younger intermediate-series biotite granite mentary-volcanic Gyeongsang basin, comprising of non-marine sedimentary and andesitic to rhyolitic volcaniclastic rocks. The Gigye granite intruded the Cretaceous sedimentary strata and may be a split-off part of the Namsan granite, ca. $20 \mathrm{~km}$ north, by right lateral strike-slip faulting (Hwang, 2004). The intrusion age of the Namsan granite is $48 \pm 2 \mathrm{Ma}$ in laser ablation ICP-MS U-Pb zircon dating (Y.J. Jwa, unpublished data), thus Paleogene.

Both the Gigye granite and the Namsan granite show very similar petrographic and geochemical characteristics (Lee et al., 1995; Koh et al., 1996; Hwang, 2004). They are mainly composed of perthite, quartz, alkaline amphibole (riebeckite and arfvedsonite), biotite (annite), and opaque minerals. Measurement of the magnetic susceptibility on the sampling sites indicates low values as 0.2 to $3.5 \times 10^{-3} \mathrm{SI}$ unit, corresponding to ilmenite series. Chemical compositions of the granites suggest that they are strongly of the A-type granites (Koh et al., 1996).

\subsection{Hapcheon syenite}

The Hapcheon syenite occurs at the southeastern margin of the exposed Yeongnam massif, and is locally covered by the Cretaceous sediments of the western Geongsang basin. This body belongs to a complex of syenite-diorite-gabbro association, and can be interpreted as a later intrusive rock than the other rock types. It is medium to coarse-grained rock and mainly 
Table 2 Polarized XRF analyses of the studied granites.

\begin{tabular}{|c|c|c|c|c|c|c|c|c|c|c|c|c|c|}
\hline$\overline{\text { Plutons }}$ & & & & Daegang & ranite & & & & & Hamc & $\overline{\text { hang Gra }}$ & רite & \\
\hline Sample nos. & R1 & R3 & R3e & R3a & R8 & R9 & R10 & $\overline{\mathrm{R} 11 \mathrm{a}}$ & R21 & R22 & R22m & R28 & R29 \\
\hline $\mathrm{SiO}_{2} \mathrm{wt}^{2} \%$ & 74.76 & 75.80 & 70.75 & 76.68 & 74.31 & 73.96 & 74.81 & 75.12 & 71.43 & 71.21 & 68.65 & 70.14 & 71.23 \\
\hline $\mathrm{TiO}_{2}$ & 0.22 & 0.11 & 0.25 & 0.09 & 0.18 & 0.19 & 0.19 & 0.03 & 0.20 & 0.28 & 0.35 & 0.27 & 0.32 \\
\hline $\mathrm{Al}_{2} \mathrm{O}_{3}$ & 12.32 & 12.39 & 14.30 & 11.99 & 12.55 & 12.73 & 12.90 & 13.96 & 14.21 & 13.85 & 15.49 & 14.61 & 13.74 \\
\hline $\mathrm{Fe}_{2} \mathrm{O}_{3}$ & 2.37 & 1.26 & 2.70 & 1.31 & 2.33 & 2.36 & 1.52 & 0.51 & 2.23 & 2.07 & 2.85 & 2.79 & 3.19 \\
\hline $\mathrm{MnO}$ & 0.03 & 0.02 & 0.04 & 0.02 & 0.04 & 0.04 & 0.02 & 0.01 & 0.04 & 0.03 & 0.04 & 0.04 & 0.05 \\
\hline $\mathrm{MgO}$ & 0.14 & 0.07 & 0.20 & 0.04 & 0.08 & 0.06 & 0.17 & 0.07 & 0.11 & 1.04 & 1.21 & 0.25 & 0.24 \\
\hline $\mathrm{CaO}$ & 0.46 & 0.47 & 0.89 & 0.40 & 0.52 & 0.58 & 0.60 & 0.74 & 1.01 & 2.15 & 2.61 & 1.06 & 1.09 \\
\hline $\mathrm{Na}_{2} \mathrm{O}$ & 3.97 & 4.13 & 5.17 & 3.93 & 4.24 & 4.21 & 3.95 & 4.42 & 3.59 & 3.65 & 4.23 & 3.64 & 3.57 \\
\hline $\mathrm{K}_{2} \mathrm{O}$ & 4.67 & 4.96 & 4.70 & 4.73 & 4.96 & 5.19 & 4.91 & 4.57 & 6.00 & 3.95 & 3.52 & 6.17 & 5.55 \\
\hline $\mathrm{P}_{2} \mathrm{O}_{5}$ & $<0.01$ & $<0.01$ & 0.02 & $<0.01$ & $<0.01$ & $<0.01$ & $<0.01$ & $<0.01$ & $<0.01$ & 0.05 & 0.08 & 0.03 & 0.05 \\
\hline $\mathrm{H}_{2} \mathrm{O}^{+}$ & 0.51 & 0.33 & 0.46 & 0.30 & 0.33 & 0.31 & 0.61 & 0.33 & 0.54 & 0.73 & 0.68 & 0.62 & 0.61 \\
\hline $\mathrm{H}_{2} \mathrm{O}^{-}$ & 0.14 & 0.15 & 0.26 & 0.13 & 0.12 & 0.13 & 0.17 & 0.15 & 0.08 & 0.19 & 0.13 & 0.25 & 0.16 \\
\hline $\mathrm{S}$ & 0.02 & 0.02 & 0.02 & 0.02 & 0.01 & 0.02 & 0.02 & 0.01 & 0.01 & 0.01 & 0.01 & 0.01 & 0.01 \\
\hline $\mathrm{CO}_{2}$ & 0.17 & 0.11 & 0.07 & 0.10 & 0.04 & 0.07 & 0.57 & 0.06 & 0.62 & 0.61 & 0.08 & 0.41 & 0.20 \\
\hline $\mathrm{F}$ & 0.18 & 0.11 & 0.35 & 0.13 & 0.11 & 0.15 & 0.11 & 0.02 & 0.06 & 0.07 & 0.06 & 0.05 & 0.06 \\
\hline Total & 99.96 & 99.93 & 100.18 & 99.87 & 99.82 & 100.00 & 100.55 & 100.00 & 100.13 & 99.89 & 99.99 & 100.34 & 100.07 \\
\hline$\overline{\mathrm{Rb} p p m}$ & 209 & 239 & 289 & 235 & 171 & 154 & 191 & 183 & 156 & 101 & 86 & 153 & 171 \\
\hline $\mathrm{Sr}$ & 29 & 25 & 26 & 14 & 11 & 13 & 70 & 57 & 77 & 459 & 545 & 88 & 98 \\
\hline $\mathrm{Ba}$ & 121 & 109 & 132 & 35 & 66 & 66 & 220 & 170 & 295 & 1180 & 1020 & 411 & 426 \\
\hline $\mathrm{Zr}$ & 476 & 184 & 336 & 53 & 464 & 457 & 159 & 22 & 268 & 71 & 114 & 326 & 413 \\
\hline $\mathrm{Hf}$ & 14.5 & 7.3 & 9.5 & 4.7 & 11.4 & 10.5 & 6.2 & 1.6 & 6.1 & 1.0 & 2.2 & 6.6 & 10.9 \\
\hline $\mathrm{Nb}$ & 26 & 15.9 & 21 & 20 & 19.6 & 17.7 & 9.4 & 6.7 & 11.6 & 2.4 & 3.6 & 12.3 & 14.8 \\
\hline $\mathrm{Ta}$ & 3.6 & 3.8 & 2.7 & 3.8 & 2.3 & 1.9 & 2.8 & 3.5 & 2.8 & 1.2 & 1.1 & 2.2 & 3.0 \\
\hline Y & 76 & 52 & 61 & 56 & 68 & 50 & 43 & 16 & 25 & 4 & 4 & 27 & 36 \\
\hline $\mathrm{La}$ & 111 & 74 & 145 & 26 & 137 & 114 & 92 & 9 & 118 & 24 & 30 & 148 & 137 \\
\hline $\mathrm{Ce}$ & 206 & 131 & 256 & 55 & 218 & 209 & 122 & 15 & 203 & 40 & 51 & 257 & 240 \\
\hline$\overline{\mathrm{V}}$ & 9 & $<2$ & 2 & 3 & $<2$ & $<2$ & $<2$ & 3 & $<3$ & 23 & 30 & $<4$ & $<4$ \\
\hline $\mathrm{Cr}$ & 12 & 12 & 10 & 6 & 9 & 19 & 9 & 24 & 11 & 36 & 36 & 11 & 13 \\
\hline Co & $<5$ & $<5$ & $<5$ & $<5$ & $<5$ & $<5$ & $<5$ & $<2$ & $<5$ & $<5$ & $<5$ & $<5$ & $<5$ \\
\hline $\mathrm{Ni}$ & 4 & 3 & 1 & 2 & 3 & 3 & 3 & 3 & 2 & 10 & 10 & 2 & 3 \\
\hline $\mathrm{Cu}$ & 0.4 & 6.3 & 0.9 & 1.2 & $<0.6$ & 1.1 & 1.0 & 0.7 & 0.3 & 1.9 & 1.5 & 0.4 & 2.4 \\
\hline $\mathrm{Zn}$ & 107 & 61 & 112 & 62 & 94 & 86 & 43 & 25 & 43 & 38 & 41 & 51 & 62 \\
\hline $\mathrm{Pb}$ & 30 & 35 & 29 & 39 & 28 & 14 & 29 & 38 & 27 & 20 & 21 & 26 & 28 \\
\hline $\mathrm{Ga}$ & 23.5 & 22.7 & 24.0 & 23.0 & 23.0 & 22.9 & 17.9 & 16.9 & 19.4 & 15.3 & 16.8 & 19.1 & 19.4 \\
\hline $\mathrm{Ge}$ & 1.1 & 1.2 & 1.5 & 1.3 & 1.2 & 1.1 & 0.9 & 1.4 & 1.0 & 0.9 & 1.0 & 1.0 & 1.1 \\
\hline $\mathrm{Se}$ & 0.4 & 0.3 & 0.3 & 0.3 & 0.2 & 0.2 & 0.3 & 0.2 & 0.2 & 0.3 & 0.2 & $<0.1$ & 1.0 \\
\hline Mo & 2.0 & 1.5 & 0.8 & 1.5 & $<0.2$ & 2.7 & $<0.2$ & $<0.2$ & 0.3 & $<0.2$ & $<0.2$ & 1.5 & 3.7 \\
\hline W & $<2$ & $<1$ & $<2$ & $<1$ & $<2$ & $<1$ & $<1$ & $<1$ & $<1$ & $<1$ & $<1$ & $<1$ & $<1$ \\
\hline $\mathrm{Sn}$ & 6.1 & 4.7 & 19.3 & 7.2 & 3.8 & 3.2 & 5.4 & 5.5 & 2.0 & 0.8 & 0.9 & 2.0 & 2.8 \\
\hline Cs & 3.0 & 8.3 & 14.7 & 5.2 & 7.4 & 7.2 & 4.0 & 5.5 & 1.4 & 4.6 & 2.9 & 4.3 & 1.9 \\
\hline $\mathrm{Tl}$ & 2.9 & 2.2 & 2.7 & 2.2 & 1.4 & 1.4 & 1.5 & 1.7 & 1.5 & 0.9 & 0.8 & 1.4 & 3.9 \\
\hline $\mathrm{Bi}$ & 1.0 & 0.7 & 0.6 & 0.4 & 0.6 & 0.4 & 0.3 & 0.6 & $<0.3$ & $<0.3$ & 0.5 & 0.3 & 1.3 \\
\hline Th & 28 & 24 & 42 & 33 & 23 & 22 & 32 & 3.7 & 28 & 12.4 & 9.3 & 26 & 32 \\
\hline $\mathrm{U}$ & 3.3 & 5.6 & 5.2 & 4.1 & 3.4 & 1.9 & 4.4 & 1.4 & 2.2 & $<0.5$ & 0.4 & $<0.5$ & 3.3 \\
\hline $\mathrm{CNK} / \mathrm{A}$ & 0.99 & 0.95 & 0.94 & 0.97 & 0.94 & 0.94 & 1.00 & 1.03 & 1.00 & 0.98 & 1.00 & 1.00 & 0.99 \\
\hline $10000 x \mathrm{xa} / \mathrm{A}$ & 3.61 & 3.46 & 3.17 & 3.63 & 3.46 & 3.40 & 2.62 & 2.29 & 2.58 & 2.09 & 2.05 & 2.47 & 2.67 \\
\hline $\mathrm{Rb} / \mathrm{Sr}$ & 7.21 & 9.56 & 11.12 & 16.79 & 15.55 & 11.85 & 2.73 & 3.21 & 2.03 & 0.22 & 0.16 & 1.74 & 1.74 \\
\hline $\mathrm{Sr} / \mathrm{Y}$ & 0.38 & 0.48 & 0.43 & 0.25 & 0.16 & 0.26 & 1.63 & 3.56 & 3.08 & 114.75 & 136.25 & 3.26 & 2.72 \\
\hline
\end{tabular}

Analyst: B. W. Chappell, GEOMOC, Sydney. F analyzed by ISE method (Actlabs. L.td)

comprises microcline, perthite, amphibole and subordinate amount of quartz. Diorite, which was intruded by the syenite, has much more amount of mafic minerals $(20-30 \%)$ and shows a foliated texture. They are classified as the magnetite series, based on measurement of the magnetic susceptibility $\left(15-30 \times 10^{-3} \mathrm{SI}\right)$. Though the radiometric ages of syenite and diorite are unknown, they are assumed to be early Jurassic on the basis of regional igneous activity around the area where the rocks are distributed (Kim and Turek, 1996).

\section{Petrography and Chemical Compositions}

Localities, rock types and magnetic susceptibility as measured by field apparatus (Kappameter KT-5) of the analyzed samples are listed in Table 1 . The analytical results are given in Tables 2 and 3 .

The Daegang granite belongs to the ilmenite series, and is rich in both $\mathrm{Na}_{2} \mathrm{O}+\mathrm{K}_{2} \mathrm{O}$ and $\mathrm{K}_{2} \mathrm{O}$, and plotted in the high-K calc-alkaline series (Fig. 3). It has very low contents of $\mathrm{CaO}$ as $0.4-0.9 \%$, and $\mathrm{Ba}$ content (35-220 
Table 2 (continued).

\begin{tabular}{|c|c|c|c|c|c|}
\hline \multirow{2}{*}{$\begin{array}{l}\text { Plutons } \\
\text { Sample nos. }\end{array}$} & \multicolumn{2}{|c|}{ Gigye Granite } & \multicolumn{3}{|c|}{ Hapcheon Syenite } \\
\hline & R30 & R31 & R33 & R34 & $\mathrm{R} 35$ \\
\hline$\overline{\mathrm{SiO}_{2} \text { wt. } \%}$ & 76.10 & 76.37 & 62.45 & 62.79 & 73.02 \\
\hline $\mathrm{TiO}_{2}$ & 0.10 & 0.11 & 0.67 & 0.48 & 0.24 \\
\hline $\mathrm{Al}_{2} \mathrm{O}_{3}$ & 11.81 & 11.59 & 16.84 & 18.19 & 13.95 \\
\hline $\mathrm{Fe}_{2} \mathrm{O}_{3}$ & 2.20 & 1.94 & 5.25 & 3.25 & 1.46 \\
\hline $\mathrm{MnO}$ & 0.05 & 0.04 & 0.14 & 0.07 & 0.03 \\
\hline $\mathrm{MgO}$ & 0.06 & 0.09 & 0.57 & 0.52 & 0.32 \\
\hline $\mathrm{CaO}$ & $<0.01$ & 0.30 & 1.21 & 1.80 & 0.65 \\
\hline $\mathrm{Na}_{2} \mathrm{O}$ & 4.56 & 4.68 & 6.98 & 6.85 & 4.13 \\
\hline $\mathrm{K}_{2} \mathrm{O}$ & 4.32 & 4.34 & 5.24 & 4.96 & 5.43 \\
\hline $\mathrm{P}_{2} \mathrm{O}_{5}$ & $<0.01$ & $<0.01$ & 0.16 & 0.17 & 0.02 \\
\hline $\mathrm{H}_{2} \mathrm{O}^{+}$ & 0.38 & 0.21 & 0.33 & 0.34 & 0.47 \\
\hline $\mathrm{H}_{2} \mathrm{O}^{-}$ & 0.19 & 0.13 & 0.19 & 0.17 & 0.18 \\
\hline $\mathrm{S}$ & $<0.01$ & $<0.01$ & $<0.01$ & $<0.01$ & $<0.01$ \\
\hline $\mathrm{CO}_{2}$ & 0.07 & 0.07 & 0.14 & 0.17 & 0.12 \\
\hline $\mathrm{F}$ & 0.05 & 0.17 & 0.04 & 0.04 & 0.03 \\
\hline Total & 99.89 & 100.04 & 100.21 & 99.80 & 100.05 \\
\hline$\overline{\mathrm{Rb} p p m}$ & 167 & 144 & 67 & 85 & 179 \\
\hline $\mathrm{Sr}$ & 9.0 & 17 & 91 & 345 & 157 \\
\hline $\mathrm{Ba}$ & 69 & 99 & 615 & 960 & 750 \\
\hline $\mathrm{Zr}$ & 514 & 396 & 106 & 235 & 223 \\
\hline $\mathrm{Hf}$ & 17.0 & 14.2 & 2.7 & 5.7 & 5.7 \\
\hline $\mathrm{Nb}$ & 39 & 28 & 1.1 & 2.6 & 6.4 \\
\hline $\mathrm{Ta}$ & 5.2 & 4.5 & 1.2 & $<2$ & 1.5 \\
\hline $\mathrm{Y}$ & 93 & 97 & 28 & 19 & 17 \\
\hline $\mathrm{La}$ & 56 & 77 & 30 & 23 & 43 \\
\hline $\mathrm{Ce}$ & 49 & 110 & 70 & 48 & 63 \\
\hline$\overline{\mathrm{V}}$ & $<2$ & 5 & $<5$ & 8 & 12 \\
\hline $\mathrm{Cr}$ & 12 & 11 & 14 & 9 & 9 \\
\hline Co & 6 & $<5$ & $<5$ & 7 & $<5$ \\
\hline $\mathrm{Ni}$ & 4 & 4 & 1 & 3 & 4 \\
\hline $\mathrm{Cu}$ & 0.6 & 1.5 & 4.1 & 2.7 & 3.1 \\
\hline $\mathrm{Zn}$ & 209 & 100 & 118 & 55 & 26 \\
\hline $\mathrm{Pb}$ & 26 & 21 & 10 & 23 & 17 \\
\hline $\mathrm{Ga}$ & 23.0 & 21.4 & 26.5 & 21.1 & 14.5 \\
\hline $\mathrm{Ge}$ & 1.9 & 1.5 & 1.6 & 1.0 & 1.0 \\
\hline $\mathrm{Se}$ & 0.4 & 0.3 & 0.3 & 0.9 & 0.6 \\
\hline Mo & 2.6 & 2.0 & 2.0 & 4.3 & 3.3 \\
\hline W & $<2$ & $<2$ & $<2$ & $<1$ & $<1$ \\
\hline Sn & 11.9 & 7.5 & 1.5 & 1.5 & 1.3 \\
\hline Cs & 2.3 & 3.2 & $<1.5$ & 2.5 & 8.5 \\
\hline $\mathrm{Tl}$ & 2.8 & 2.4 & 1.2 & 3.5 & 2.7 \\
\hline $\mathrm{Bi}$ & 1.0 & 0.9 & 0.4 & 1.7 & 1.4 \\
\hline Th & 24 & 18.9 & 0.6 & 6.0 & 27 \\
\hline $\mathrm{U}$ & 8.0 & 4.9 & 0.6 & 5.3 & 8.5 \\
\hline$\overline{\mathrm{CNK} / \mathrm{A}}$ & 0.97 & 0.90 & 0.87 & 0.91 & 1.01 \\
\hline $10000 x G a / A$ & 3.68 & 3.49 & 2.97 & 2.19 & 1.96 \\
\hline $\mathrm{Rb} / \mathrm{Sr}$ & 18.56 & 8.47 & 0.74 & 0.25 & 1.14 \\
\hline $\mathrm{Sr} / \mathrm{Y}$ & 0.10 & 0.18 & 3.25 & 18.16 & 9.24 \\
\hline
\end{tabular}

ppm) and Sr content (11-70 ppm) are also low, reflecting one feldspar characteristic of the granite. The CNK/ A ratio is below 1, which is meta-aluminous, except for a minor phase of the leucogranite (R11). The Ga contents (18-24 ppm) are not high, but Ga/Al x 10,000 value is higher than 2.6 (Fig. 4), thus plotted in the Atype field (Wahlen et al., 1982), because of relatively low $\mathrm{Al}_{2} \mathrm{O}_{3}$ contents.

The Hamchang granite also belongs to ilmenite series (Table 1). The analyzed samples are divided into
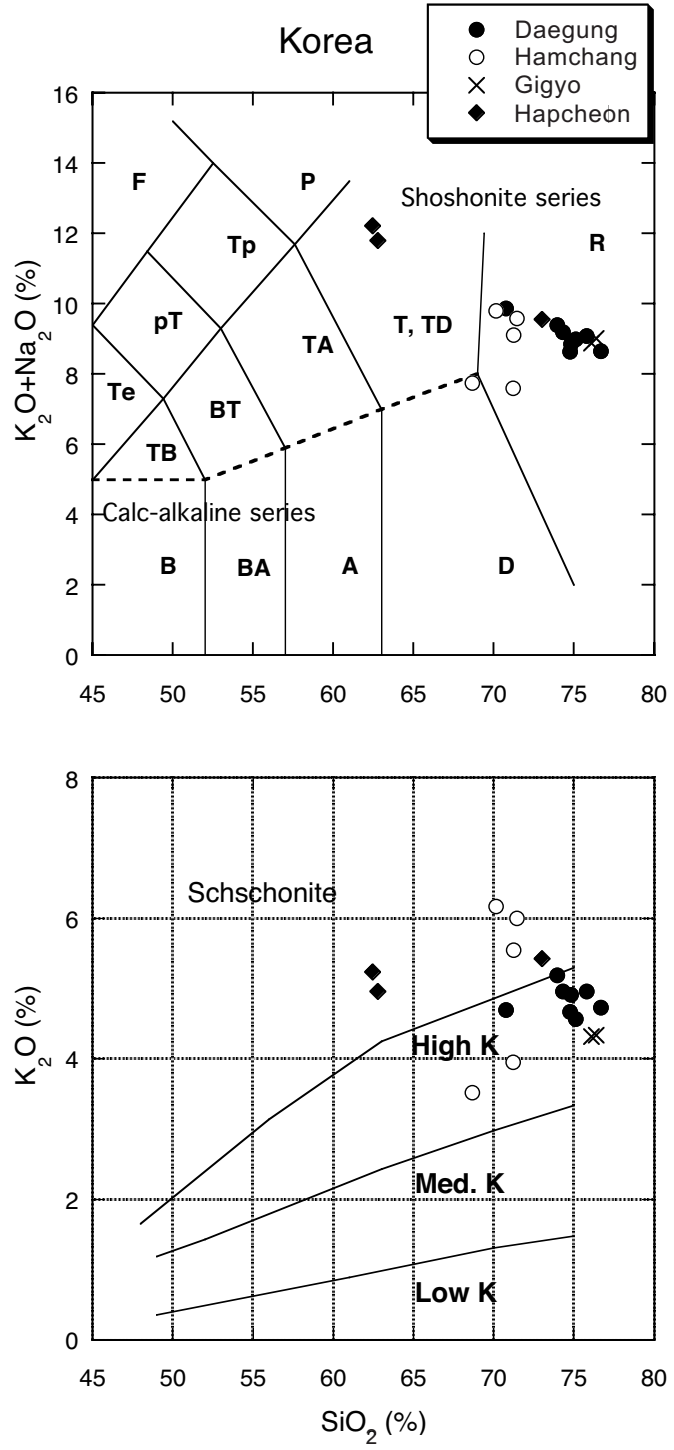

Fig. 3 Total alkalis vs. silica and potassium vs. silica contents of the studied granites. The classification based upon Le Maitre et al. (1989). Abbreviation: D, dacite, R, rhyolite; T, trachyte (qz<20\%), TD, trachydacite ( $\mathrm{qz}>20 \%)$.

two groups on the alkali contents; high-alkali group is plotted in the shoshonite field, but the low one in the high-K calc-alkaline field (Fig. 3). Alumina saturation of the two groups is almost the same, CNK/A ratio around 1 , but the low alkali group has very high contents of $\mathrm{Sr}$ (459-545 ppm) and $\mathrm{Ba}(1,020-1,180 \mathrm{ppm})$, and low contents of Y (4 ppm). The obtained high $\mathrm{Sr} /$ Y ratios (115-136, Table 2) are suggestive for adakitic nature coexisting with shoshonite-series granites. The high $\mathrm{Sr} / \mathrm{Y}$ granites have been known in the Jecheon pluton, though the age is Triassic-Jurassic, to $50 \mathrm{~km}$ north of this body (Ishihara et al., 2005).

The Gigye granite of the studied two samples belong to ilmenite series and high-K series (Fig. 3). $\mathrm{CaO}$ 
Table 3 ICP-ICP/MS analyses of the studied granites.

\begin{tabular}{|c|c|c|c|c|c|c|c|c|c|c|c|c|c|}
\hline \multirow[b]{2}{*}{ Element: } & \multicolumn{8}{|c|}{ Daegang granite } & \multicolumn{5}{|c|}{ Hamchang granite } \\
\hline & R1 & R3 & R3e & R3a & R8 & R9 & R10 & R11a & R21 & R22 & $\mathrm{R} 22 \mathrm{~m}$ & $\mathrm{R} 28$ & R29 \\
\hline $\mathrm{Rb}$ & 204 & 234 & 299 & 232 & 172 & 153 & 194 & 196 & 167 & 104 & 94 & 162 & 185 \\
\hline $\mathrm{Sr}$ & 27 & 24 & 27 & 15 & 13 & 15 & 68 & 58 & 78 & 423 & 515 & 87 & 94 \\
\hline $\mathrm{Ba}$ & 124 & 106 & 135 & 35 & 63 & 64 & 230 & 190 & 323 & 1230 & 1120 & 438 & 480 \\
\hline Cs & 3.7 & 4.7 & 12.9 & 3.8 & 4.0 & 6.1 & 2.1 & 5.4 & 1.7 & 2.9 & 2.6 & 2.2 & 2.7 \\
\hline Ga & 27 & 25 & 28 & 26 & 27 & 26 & 21 & 20 & 23 & 18 & 20 & 24 & 25 \\
\hline $\mathrm{Ge}$ & 1.7 & 1.5 & 1.9 & 1.5 & 1.6 & 1.4 & 1.3 & 1.7 & 1.3 & 0.9 & 1 & 1.3 & 1.6 \\
\hline $\mathrm{La}$ & 94.9 & 57.2 & 121 & 18.7 & 115 & 90.9 & 74.3 & 7.85 & 102 & 34.4 & 34.5 & 124 & 130 \\
\hline $\mathrm{Ce}$ & 183 & 110 & 227 & 44.7 & 192 & 175 & 106 & 14.9 & 186 & 58.7 & 58.2 & 229 & 237 \\
\hline $\mathrm{Pr}$ & 21.5 & 12.5 & 25.1 & 6.07 & 24.4 & 20.5 & 14.5 & 1.86 & 21.2 & 6.24 & 6.08 & 26.4 & 27.7 \\
\hline $\mathrm{Nd}$ & 72.9 & 41.4 & 76.1 & 27.1 & 79.4 & 66.9 & 43.7 & 7.66 & 64.9 & 19 & 18.5 & 81.6 & 87 \\
\hline $\mathrm{Sm}$ & 14.1 & 8.15 & 13.1 & 6.47 & 15.2 & 12.7 & 8.19 & 1.92 & 9.79 & 2.72 & 2.68 & 12.1 & 13.8 \\
\hline $\mathrm{Eu}$ & 0.392 & 0.266 & 0.403 & 0.132 & 0.206 & 0.188 & 0.56 & 0.221 & 0.869 & 0.617 & 0.775 & 1.11 & 1.13 \\
\hline LREE & 386.79 & 229.52 & 462.70 & 103.17 & 426.21 & 366.19 & 247.25 & 34.41 & 384.76 & 121.68 & 120.74 & 474.21 & 496.63 \\
\hline $\mathrm{Gd}$ & 12.9 & 7.61 & 10.1 & 7.17 & 14.0 & 10.6 & 7.56 & 2.19 & 6.79 & 1.77 & 1.72 & 7.82 & 9.32 \\
\hline $\mathrm{Tb}$ & 2.34 & 1.48 & 1.86 & 1.49 & 2.24 & 1.77 & 1.3 & 0.49 & 0.98 & 0.25 & 0.26 & 1.11 & 1.42 \\
\hline Dy & 13.6 & 8.71 & 10.3 & 9.43 & 12.0 & 9.67 & 7.3 & 3.17 & 5.11 & 1.34 & 1.39 & 5.69 & 7.29 \\
\hline Но & 2.71 & 1.78 & 2.06 & 1.92 & 2.34 & 1.84 & 1.45 & 0.6 & 0.95 & 0.25 & 0.25 & 1.05 & 1.38 \\
\hline $\mathrm{Er}$ & 8.3 & 5.62 & 6.48 & 6.04 & 7.15 & 5.8 & 4.63 & 1.87 & 2.9 & 0.76 & 0.76 & 3.16 & 4.2 \\
\hline $\mathrm{Tm}$ & 1.25 & 0.901 & 1.06 & 0.925 & 1.1 & 0.881 & 0.739 & 0.307 & 0.446 & 0.114 & 0.114 & 0.461 & 0.636 \\
\hline $\mathrm{Yb}$ & 7.48 & 5.44 & 6.56 & 5.84 & 6.59 & 5.52 & 4.66 & 1.97 & 2.74 & 0.75 & 0.8 & 2.67 & 3.86 \\
\hline $\mathrm{Lu}$ & 1.02 & 0.733 & 0.925 & 0.761 & 0.955 & 0.796 & 0.635 & 0.275 & 0.381 & 0.104 & 0.114 & 0.38 & 0.548 \\
\hline HREE & 49.60 & 32.27 & 39.35 & 33.58 & 46.38 & 36.88 & 28.27 & 10.87 & 20.30 & 5.34 & 5.41 & 22.34 & 28.65 \\
\hline Y & 74.6 & 51.5 & 61.7 & 55.6 & 69.2 & 51.5 & 43.1 & 20.4 & 27.7 & 7.3 & 8.1 & 29.6 & 39.6 \\
\hline HREE+Y & 124.20 & 83.77 & 101.05 & 89.18 & 115.58 & 88.38 & 71.37 & 31.27 & 48.00 & 12.64 & 13.51 & 51.94 & 68.25 \\
\hline $\mathrm{Zr}$ & 612 & 250 & 476 & 73 & 622 & 642 & 225 & 30 & 362 & 106 & 152 & 434 & 624 \\
\hline $\mathrm{Hf}$ & 14.9 & 7.4 & 12.8 & 3.5 & 13.4 & 13.3 & 6.6 & 1.1 & 8.2 & 2.7 & 3.5 & 9.3 & 13.2 \\
\hline $\mathrm{Nb}$ & 28.4 & 19 & 24.9 & 23.5 & 23.9 & 21.5 & 13.3 & 10.6 & 16.5 & 6.3 & 8 & 16 & 20.4 \\
\hline $\mathrm{Ta}$ & 2.03 & 1.7 & 2 & 2.32 & 1.45 & 1.26 & 1.33 & 1.97 & 1.17 & 0.37 & 0.57 & 0.97 & 1.28 \\
\hline $\mathrm{Sn}$ & 4 & 4 & 17 & 6 & 3 & 2 & 4 & 5 & 1 & $<1$ & $<1$ & 1 & 2 \\
\hline W & 1.1 & 1 & 1.3 & 1.6 & 0.6 & 0.5 & 1.1 & $<0.5$ & 2.7 & 0.6 & 0.7 & 0.7 & $<0.5$ \\
\hline $\mathrm{Tl}$ & 1.5 & 1.69 & 2.17 & 1.66 & 1.1 & 0.99 & 1.32 & 1.27 & 1 & 0.69 & 0.55 & 0.97 & 1.19 \\
\hline $\mathrm{Pb}$ & 30 & 36 & 30 & 44 & 24 & 16 & 32 & 40 & 28 & 23 & 20 & 28 & 31 \\
\hline $\mathrm{Sb}$ & 1.2 & 1.6 & 1.2 & 1.5 & 1.3 & 0.9 & 1.4 & 0.9 & 1.2 & 1.7 & 1 & 1.4 & 1.6 \\
\hline Th & 27.9 & 24.4 & 43.5 & 33.3 & 25.8 & 22.3 & 32.4 & 6.62 & 29.3 & 13.2 & 10.3 & 29.2 & 38.3 \\
\hline $\mathrm{U}$ & 4.17 & 5.64 & 5.8 & 4.22 & 3.61 & 3.34 & 5.5 & 3.51 & 2.91 & 0.94 & 1.47 & 2.27 & 3.36 \\
\hline $\mathrm{Rb} / \mathrm{Sr}$ & 7.6 & 9.8 & 11.1 & 15.5 & 13.2 & 1 & 2.9 & 3.4 & 2.2 & 0.3 & 0.2 & 1.9 & 2 \\
\hline $\mathrm{Sr} / \mathrm{Y}$ & 0.4 & 0.5 & 0.4 & 0.3 & 0.2 & 0.3 & 1.6 & 2.8 & 2.8 & 58 & 63.6 & 2.9 & 2.4 \\
\hline L/HREE & 7.8 & 7.1 & 11.8 & 3.1 & 9.2 & 9.9 & 8.8 & 3.2 & 18.5 & 22.8 & 22.3 & 21.2 & 17.3 \\
\hline REE & 436.4 & 261.8 & 502.1 & 136.8 & 472.6 & 403.1 & 275.5 & 45.3 & 405.1 & 127.0 & 126.2 & 496.6 & 525.3 \\
\hline REE $+\mathrm{Y}$ & 511.0 & 313.3 & 563.8 & 192.4 & 541.8 & 454.6 & 318.6 & 65.7 & 432.8 & 134.3 & 134.3 & 526.2 & 564.9 \\
\hline
\end{tabular}

Analyses: ICP-ICP/MS by Actlabs., Ltd. The contents in ppm

is very low, so that $\mathrm{Ba}(69-99 \mathrm{ppm})$ and $\mathrm{Sr}(9-17 \mathrm{ppm})$ are low. $\mathrm{Zr}$ (396-514 ppm), Zn (100-209 ppm), Nb (28-39 ppm) and Y (93-97 ppm) are high. The alumina saturation index is less than unity (Fig. 4).

The Hapcheon syenite belongs to magnetite series (Table 1) and shoshonite series in the alkali-silica plots (Fig. 3) and is meta-aluminous. The sample R33 reveals a high $\mathrm{Ga} / \mathrm{Al} \times 10,000$ value as 3.0 , while the others are lower than 2.2 (Fig. 4).

\section{REE Contents of the Granitoids}

About the Daegang granite, Kim et al. (1998) reported REE contents on 8 samples, and revealed the total REE varying from 301 to $662 \mathrm{ppm}$ with L/HREE ratio of 5.3 to 13.1 for the normal phase $(\mathrm{n}=5)$, and 511 to $1,082 \mathrm{ppm}$ with L/HREE ratio of 10.9 to 36.0 for the contained enclaves. Thus the enclaves are enriched in REE, especially of HREE, and the bulk contents are much depend on the amount of LREE. 
Table 3 (continued)

\begin{tabular}{|c|c|c|c|c|c|}
\hline \multicolumn{3}{|c|}{ Gigye granite } & \multicolumn{3}{|c|}{ Hapcheon syenite } \\
\hline Element: & $\mathrm{R} 30$ & $\mathrm{R} 31$ & $\mathrm{R} 33$ & $\mathrm{R} 34$ & $\mathrm{R} 35$ \\
\hline $\mathrm{Rb}$ & 164 & 143 & 65 & 87 & 189 \\
\hline $\mathrm{Sr}$ & 8 & 16 & 85 & 331 & 156 \\
\hline $\mathrm{Ba}$ & 70 & 104 & 677 & 1030 & 792 \\
\hline $\mathrm{Cs}$ & 1.5 & 1.5 & 0.5 & 1.4 & 1.7 \\
\hline $\mathrm{Ga}$ & 26 & 25 & 28 & 24 & 18 \\
\hline $\mathrm{Ge}$ & 2.2 & 2.3 & 1.5 & 1.3 & 1.1 \\
\hline $\mathrm{La}$ & 41.3 & 59.3 & 32.6 & 31.9 & 45.2 \\
\hline $\mathrm{Ce}$ & 35.3 & 93.6 & 73.5 & 60.3 & 77 \\
\hline $\operatorname{Pr}$ & 12.4 & 15.9 & 10.3 & 7.29 & 7.94 \\
\hline $\mathrm{Nd}$ & 42.4 & 57.8 & 39.1 & 27.1 & 24.2 \\
\hline $\mathrm{Sm}$ & 11 & 13.2 & 8.04 & 5.41 & 4.01 \\
\hline $\mathrm{Eu}$ & 0.289 & 0.375 & 1.77 & 2.59 & 0.676 \\
\hline LREE & 142.69 & 240.18 & 165.31 & 134.59 & 159.03 \\
\hline $\mathrm{Gd}$ & 11.9 & 14.2 & 6.88 & 4.51 & 2.76 \\
\hline $\mathrm{Tb}$ & 2.34 & 2.73 & 1.08 & 0.74 & 0.49 \\
\hline Dy & 14.2 & 16 & 5.7 & 3.98 & 2.72 \\
\hline Ho & 3.02 & 3.24 & 1.07 & 0.77 & 0.55 \\
\hline $\mathrm{Er}$ & 10.2 & 10.2 & 3.12 & 2.24 & 1.79 \\
\hline $\mathrm{Tm}$ & 1.83 & 1.58 & 0.443 & 0.32 & 0.286 \\
\hline $\mathrm{Yb}$ & 11.8 & 10 & 2.75 & 2.05 & 1.86 \\
\hline $\mathrm{Lu}$ & 1.73 & 1.42 & 0.4 & 0.306 & 0.281 \\
\hline HREE & 57.02 & 59.37 & 21.44 & 14.92 & 10.74 \\
\hline $\mathrm{Y}$ & 88.5 & 95.7 & 29.7 & 21.7 & 17.7 \\
\hline HREE+Y & 145.52 & 155.07 & 51.14 & 36.62 & 28.44 \\
\hline $\mathrm{Zr}$ & 646 & 483 & 175 & 305 & 273 \\
\hline $\mathrm{Hf}$ & 19.4 & 12.8 & 3.3 & 5.9 & 6.1 \\
\hline $\mathrm{Nb}$ & 37.3 & 31 & 6 & 5.3 & 9.7 \\
\hline $\mathrm{Ta}$ & 3.23 & 2.42 & 0.28 & 0.36 & 0.79 \\
\hline $\mathrm{Sn}$ & 10 & 7 & $<1$ & $<1$ & $<1$ \\
\hline W & 1.1 & 4 & 3.1 & $<0.5$ & $<0.5$ \\
\hline $\mathrm{Tl}$ & 1.32 & 0.85 & 0.38 & 0.5 & 0.76 \\
\hline $\mathrm{Pb}$ & 29 & 24 & 11 & 19 & 12 \\
\hline $\mathrm{Sb}$ & 1.4 & 1.6 & 1.5 & 1.3 & 1.3 \\
\hline $\mathrm{Th}$ & 25.4 & 20.1 & 1.36 & 5.95 & 26.5 \\
\hline $\mathrm{U}$ & 7.47 & 4.8 & 0.47 & 1.76 & 2.92 \\
\hline $\mathrm{Rb} / \mathrm{Sr}$ & 20.5 & 8.9 & 0.8 & 0.3 & 1.2 \\
\hline $\mathrm{Sr} / \mathrm{Y}$ & 0.1 & 0.2 & 2.9 & 15.3 & 8.8 \\
\hline L/HREE & 2.5 & 4.1 & 7.7 & 9 & 14.8 \\
\hline REE & 199.7 & 299.5 & 186.8 & 149.5 & 169.8 \\
\hline $\mathrm{REE}+\mathrm{Y}$ & 288.2 & 395.2 & 216.5 & 171.2 & 187.5 \\
\hline
\end{tabular}

Our results are lower than these values. Except for the low values of aplitic granite (R3a) and leucogranite (R11a), our REE data on 5 samples vary from $262 \mathrm{ppm}$ to $473 \mathrm{ppm}$ with the L/HREE ratio from 7.1 to 9.9 . One enclave (R3e) is also slightly higher in REE (502 ppm, L/HREE=11.8) than the normal phase (Table 3).

Fig. $4 \mathrm{CNK} / \mathrm{A}\left(\left(\mathrm{CaO}+\mathrm{Na}_{2} \mathrm{O}+\mathrm{K}_{2} \mathrm{O}\right) / \mathrm{Al}_{2} \mathrm{O}_{3}\right.$, molar ratio $), 10,000 \mathrm{Ga} /$ $\mathrm{Al}$, LREE and HREE of the studied granites.
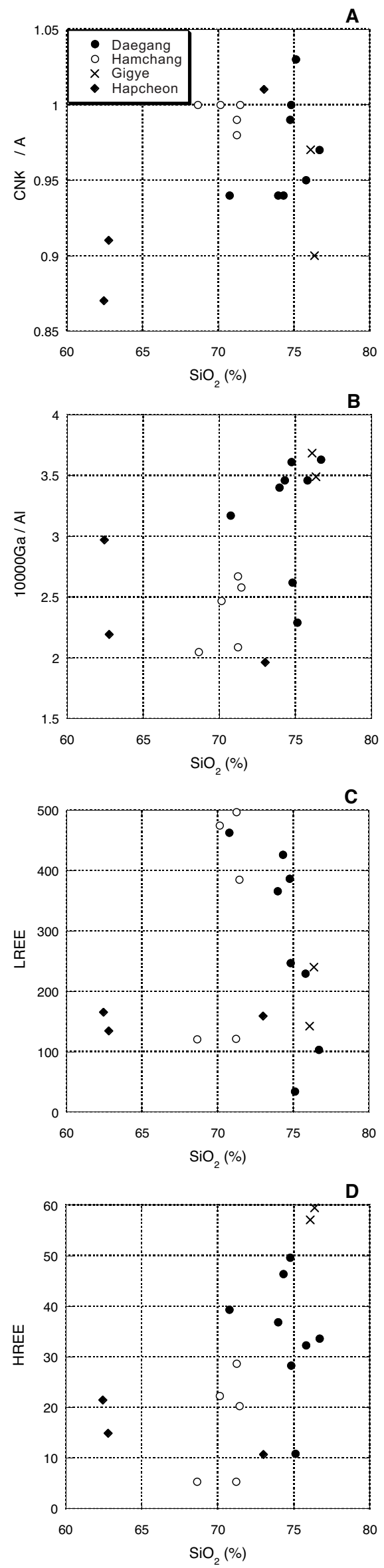
Table 4 XRF and ICP-ICP/MS analyses of the weathered soil from South Korea.

\begin{tabular}{|c|c|c|c|c|c|c|c|c|c|c|c|c|c|c|c|c|}
\hline \multicolumn{17}{|c|}{ Daegang Granite } \\
\hline Element: & K1B & K2B & K4B & K5B1 & K5B2 & K6C & K7B & K10B & K12B & K13B & $\mathrm{K} 13 \mathrm{C}$ & K14B & K16B & K17B & K18B & K19B \\
\hline $\mathrm{SiO}_{2}$ & 70.34 & 69.20 & 68.01 & 67.33 & 69.59 & 62.46 & 62.00 & 71.94 & 67.24 & 61.61 & 60.81 & 72.92 & 63.57 & 68.67 & 68.26 & 68.19 \\
\hline $\mathrm{TiO}_{2}$ & 0.69 & 0.29 & 0.36 & 0.89 & 0.94 & 0.50 & 0.95 & 0.46 & 0.69 & 0.66 & 0.74 & 0.57 & 0.67 & 0.44 & 0.81 & 0.40 \\
\hline $\mathrm{Al}_{2} \mathrm{O}_{3}$ & 13.68 & 16.40 & 15.83 & 13.74 & 13.91 & 19.73 & 15.86 & 13.48 & 15.30 & 19.25 & 18.56 & 12.95 & 17.51 & 14.95 & 15.07 & 17.40 \\
\hline $\mathrm{Fe}_{2} \mathrm{O}_{3}$ & 3.90 & 3.48 & 3.62 & 4.57 & 4.70 & 4.14 & 6.42 & 3.00 & 3.70 & 5.26 & 5.23 & 3.35 & 4.89 & 3.25 & 4.22 & 2.61 \\
\hline $\mathrm{MnO}$ & 0.07 & 0.02 & 0.01 & 0.04 & 0.04 & 0.04 & 0.12 & 0.02 & 0.06 & 0.04 & 0.06 & 0.03 & 0.06 & 0.03 & 0.05 & 0.02 \\
\hline $\mathrm{MgO}$ & 0.94 & 0.30 & 0.31 & 1.09 & 1.13 & 0.75 & 1.52 & 0.44 & 0.65 & 1.21 & 1.80 & 0.47 & 0.88 & 0.57 & 1.21 & 0.66 \\
\hline $\mathrm{CaO}$ & 0.18 & 0.04 & 0.03 & 0.08 & 0.09 & 0.26 & 0.91 & 0.13 & 0.07 & 0.62 & 1.80 & 0.06 & 0.06 & 0.15 & 0.43 & 0.54 \\
\hline $\mathrm{Na}_{2} \mathrm{O}$ & 1.33 & 0.56 & 1.15 & 0.47 & 0.51 & 0.20 & 1.61 & 1.18 & 0.20 & 0.28 & 1.09 & 0.67 & 0.20 & 0.74 & 0.70 & 1.42 \\
\hline $\mathrm{K}_{2} \mathrm{O}$ & 3.22 & 4.70 & 4.14 & 2.19 & 2.27 & 2.91 & 3.77 & 4.26 & 3.25 & 2.93 & 2.64 & 3.54 & 3.22 & 4.86 & 2.29 & 3.72 \\
\hline $\mathrm{P}_{2} \mathrm{O}_{5}$ & 0.03 & 0.01 & 0.02 & 0.02 & 0.02 & 0.03 & 0.07 & 0.02 & 0.05 & 0.03 & 0.03 & 0.02 & 0.03 & 0.03 & 0.03 & 0.02 \\
\hline $\mathrm{Cr}_{2} \mathrm{O}_{3}$ & 0.01 & $<0.01$ & $<0.01$ & 0.01 & 0.01 & $<0.01$ & $<0.01$ & $<0.01$ & $<0.01$ & $<0.01$ & $<0.01$ & $<0.01$ & $<0.01$ & $<0.01$ & $<0.01$ & $<0.01$ \\
\hline LOI & 5.01 & 5.31 & 5.42 & 8.48 & 6.35 & 8.42 & 6.47 & 4.65 & 7.46 & 8.17 & 6.95 & 4.96 & 8.99 & 5.18 & 6.53 & 5.44 \\
\hline Total & 99.40 & 100.31 & 98.90 & 98.91 & 99.56 & 99.44 & 99.70 & 99.58 & 98.67 & 100.06 & 99.71 & 99.54 & 100.08 & 98.87 & 99.60 & 100.42 \\
\hline $\mathrm{Rb}$ & 142 & 191 & 172 & 114 & 114 & 105 & 136 & 154 & 125 & 149 & 119 & 132 & 168 & 257 & 98 & 132 \\
\hline $\mathrm{Sr}$ & 47 & 18 & 14 & 46 & 47 & 74 & 96 & 40 & 75 & 71 & 214 & 28 & 43 & 42 & 87 & 180 \\
\hline $\mathrm{Ba}$ & 363 & 154 & 127 & 388 & 414 & 840 & 587 & 253 & 795 & 563 & 682 & 241 & 469 & 373 & 613 & 966 \\
\hline $\mathrm{Zr}$ & 392 & 825 & 455 & 343 & 361 & 162 & 421 & 557 & 262 & 223 & 168 & 525 & 250 & 313 & 268 & 152 \\
\hline $\mathrm{Hf}$ & 11.8 & 22.6 & 13.6 & 9.5 & 10.1 & 4.8 & 10.5 & 13.7 & 7.2 & 6.4 & 4.8 & 13.5 & 7.4 & 9.4 & 7.9 & 4.5 \\
\hline $\mathrm{Nb}$ & 24.6 & 31.2 & 32.1 & 21.5 & 22.2 & 9.2 & 17 & 19.1 & 16.3 & 13.1 & 9.3 & 25.2 & 16 & 16.7 & 16.8 & 8.6 \\
\hline $\mathrm{Ta}$ & 2.17 & 2.42 & 2.63 & 1.67 & 1.72 & 1.27 & 1.34 & 2.01 & 1.76 & 1.45 & 1.51 & 2.23 & 1.65 & 2.28 & 1.57 & 1.38 \\
\hline V & 62 & 20 & 33 & 90 & 90 & 62 & 75 & 37 & 61 & 81 & 85 & 42 & 71 & 30 & 71 & 31 \\
\hline $\mathrm{Cr}$ & 40 & $<20$ & 20 & 60 & 60 & $<20$ & 40 & 20 & 40 & 30 & 40 & 30 & 40 & $<20$ & 50 & $<20$ \\
\hline Co & 41 & 50 & 49 & 26 & 32 & 50 & 48 & 51 & 41 & 30 & 47 & 50 & 25 & 38 & 38 & 55 \\
\hline $\mathrm{Ni}$ & $<20$ & $<20$ & $<20$ & 20 & 20 & $<20$ & $<20$ & $<20$ & $<20$ & $<20$ & $<20$ & $<20$ & $<20$ & $<20$ & $<20$ & $<20$ \\
\hline $\mathrm{Cu}$ & $<10$ & $<10$ & $<10$ & 10 & 10 & $<10$ & 20 & 10 & $<10$ & $<10$ & $<10$ & $<10$ & 10 & $<10$ & $<10$ & $<10$ \\
\hline $\mathrm{Zn}$ & 70 & 80 & 60 & 60 & 50 & 50 & 50 & 50 & 40 & 70 & 60 & 60 & 50 & 40 & 60 & 50 \\
\hline $\mathrm{Pb}$ & 24 & 26 & 19 & 22 & 20 & 29 & 15 & 18 & 20 & 21 & 22 & 19 & 24 & 26 & 23 & 25 \\
\hline $\mathrm{Ga}$ & 21 & 31 & 30 & 19 & 19 & 24 & 23 & 22 & 21 & 27 & 23 & 21 & 21 & 20 & 18 & 23 \\
\hline $\mathrm{Ge}$ & 1.5 & 1.4 & 1.3 & 1.6 & 1.6 & 1.4 & 1.2 & 1.4 & 1.2 & 1.6 & 1.4 & 1.6 & 1.6 & 1.5 & 1.5 & 1.1 \\
\hline As & 8 & $<5$ & $<5$ & 11 & 10 & 5 & $<5$ & $<5$ & $<5$ & $<5$ & $<5$ & $<5$ & 10 & $<5$ & 6 & $<5$ \\
\hline Mo & $<2$ & $<2$ & $<2$ & $<2$ & $<2$ & $<2$ & $<2$ & $<2$ & $<2$ & $<2$ & $<2$ & $<2$ & $<2$ & $<2$ & $<2$ & $<2$ \\
\hline Sn & 5 & 5 & 5 & 4 & 3 & 2 & 5 & 7 & 4 & 3 & 2 & 4 & 5 & 9 & 3 & 2 \\
\hline $\mathrm{Sb}$ & 0.8 & 0.3 & 0.3 & 1.1 & 0.9 & 0.5 & 0.3 & 0.6 & 0.4 & 0.5 & 0.5 & 0.6 & 0.7 & 0.5 & 1 & 0.5 \\
\hline Cs & 4.8 & 5.4 & 4 & 6.5 & 6.5 & 4.8 & 7.1 & 6.9 & 7.9 & 11.6 & 11.3 & 5 & 9.7 & 10.3 & 6.2 & 5.9 \\
\hline $\mathrm{Tl}$ & 0.99 & 1.31 & 1.1 & 0.86 & 0.83 & 0.79 & 0.81 & 1.04 & 0.92 & 1.04 & 0.76 & 0.9 & 1.22 & 1.67 & 0.79 & 0.99 \\
\hline $\mathrm{Bi}$ & 0.2 & 0.2 & 0.2 & 0.2 & 0.2 & 0.3 & $<0.1$ & $<0.1$ & $<0.1$ & 0.3 & 0.3 & 0.2 & 0.4 & 0.1 & 0.1 & 0.1 \\
\hline Th & 17.7 & 30.4 & 29.9 & 15.7 & 15.1 & 13 & 15.8 & 16.5 & 13.9 & 20.1 & 17 & 19.1 & 23.3 & 37.8 & 14.4 & 13.8 \\
\hline $\mathrm{U}$ & 3.9 & 5.25 & 4.77 & 3.53 & 3.66 & 5.14 & 2.78 & 3.11 & 2.93 & 3.95 & 3.89 & 3.6 & 4.7 & 7.37 & 3.47 & 2.37 \\
\hline $\mathrm{La}$ & 32.1 & 73.0 & 48.0 & 40.0 & 41.6 & 41.5 & 52.5 & 42.3 & 40.5 & 39.2 & 33.0 & 39.7 & 56.7 & 42.7 & 30.8 & 30.9 \\
\hline $\mathrm{Ce}$ & 85.9 & 202.0 & 179.0 & 86.5 & 84.6 & 84.6 & 135.0 & 117.0 & 88.2 & 89.4 & 63.6 & 102.0 & 93.1 & 122.0 & 69.0 & 69.6 \\
\hline $\operatorname{Pr}$ & 6.8 & 16.0 & 10.6 & 8.3 & 8.9 & 9.0 & 11.0 & 8.3 & 7.7 & 7.5 & 7.5 & 8.4 & 11.2 & 8.4 & 6.6 & 6.0 \\
\hline $\mathrm{Nd}$ & 22.8 & 52.4 & 34.8 & 28.0 & 29.3 & 30.4 & 36.1 & 25.8 & 24.4 & 24.3 & 25.7 & 27.9 & 38.2 & 26.7 & 22.5 & 19.8 \\
\hline $\mathrm{Sm}$ & 4.26 & 9.71 & 6.99 & 5.02 & 5.29 & 5.50 & 6.32 & 4.51 & 3.99 & 3.98 & 4.43 & 5.19 & 6.22 & 4.64 & 4.04 & 3.07 \\
\hline $\mathrm{Eu}$ & 0.65 & 0.38 & 0.42 & 0.99 & 1.04 & 1.11 & 1.10 & 0.45 & 0.89 & 0.97 & 1.23 & 0.56 & 0.96 & 0.53 & 0.84 & 0.67 \\
\hline LREE & 152.5 & 353.5 & 279.8 & 168.8 & 170.7 & 172.1 & 242.0 & 198.4 & 165.6 & 165.4 & 135.4 & 183.8 & 206.4 & 205.0 & 133.7 & 130.0 \\
\hline $\mathrm{Gd}$ & 4.59 & 10.00 & 7.19 & 4.26 & 4.69 & 4.53 & 4.82 & 3.93 & 3.37 & 2.99 & 2.86 & 4.36 & 4.74 & 3.49 & 3.07 & 1.77 \\
\hline $\mathrm{Tb}$ & 0.92 & 1.85 & 1.50 & 0.79 & 0.85 & 0.72 & 0.93 & 0.84 & 0.55 & 0.54 & 0.47 & 0.97 & 0.95 & 0.68 & 0.54 & 0.26 \\
\hline Dy & 6.00 & 11.10 & 8.77 & 4.38 & 4.80 & 3.53 & 5.03 & 4.92 & 3.17 & 3.07 & 2.64 & 5.93 & 5.42 & 4.12 & 3.24 & 1.27 \\
\hline Ho & 1.28 & 2.35 & 1.80 & 0.87 & 0.95 & 0.64 & 1.03 & 1.03 & 0.65 & 0.60 & 0.50 & 1.23 & 1.02 & 0.87 & 0.68 & 0.22 \\
\hline Er & 4.26 & 7.86 & 6.17 & 2.92 & 3.15 & 1.96 & 3.37 & 3.45 & 2.08 & 1.84 & 1.47 & 4.16 & 1.18 & 2.90 & 2.09 & 0.65 \\
\hline $\mathrm{Tm}$ & 0.67 & 1.22 & 1.01 & 0.46 & 0.49 & 0.28 & 0.50 & 0.55 & 0.32 & 0.29 & 0.22 & 0.68 & 0.52 & 0.47 & 0.32 & 0.10 \\
\hline $\mathrm{Yb}$ & 4.16 & 7.72 & 5.99 & 2.89 & 3.11 & 1.76 & 3.08 & 3.50 & 2.02 & 1.80 & 1.39 & 4.24 & 3.23 & 2.90 & 2.05 & 0.63 \\
\hline $\mathrm{Lu}$ & 0.60 & 1.10 & 0.84 & 0.41 & 0.44 & 0.25 & 0.45 & 0.52 & 0.30 & 0.26 & 0.20 & 0.59 & 0.44 & 0.44 & 0.30 & 0.09 \\
\hline HREE & 22.47 & 43.20 & 33.27 & 16.99 & 18.47 & 13.67 & 19.21 & 18.74 & 12.45 & 11.38 & 9.75 & 22.16 & 17.50 & 15.86 & 12.28 & 4.99 \\
\hline $\mathrm{Y}$ & 38.7 & 69.0 & 55.0 & 27.7 & 30.2 & 18.9 & 29.1 & 31.6 & 20.2 & 19.2 & 15.7 & 39.3 & 33.0 & 26.8 & 20.5 & 7.4 \\
\hline L/HREE & 2.5 & 3.2 & 3.2 & 6.2 & 5.7 & 5.3 & 5.0 & 3.9 & 5.1 & 5.4 & 5.3 & 3.0 & 4.1 & 4.8 & 4.1 & 10.6 \\
\hline REE & 152.5 & 396.7 & 313.1 & 185.8 & 189.2 & 185.8 & 261.2 & 217.1 & 178.1 & 176.8 & 145.2 & 206.0 & 223.9 & 220.9 & 146.0 & 135.0 \\
\hline REE+Y & 191.2 & 465.7 & 368.1 & 213.5 & 219.4 & 204.7 & 290.3 & 248.7 & 198.3 & 196.0 & 160.9 & 245.3 & 256.9 & 247.7 & 166.5 & 142.4 \\
\hline
\end{tabular}

Analvst: Actlabs, Ltd. B and C attached to the sample numbers implving the soil from B horizon and C horizon.

The REE pattern of the granite (Fig. 5) indicates that the R11 leucogranite has the least values on most of the elements except for $\mathrm{Eu}$, although it is partly pegmatitic. The R10 granite is depleted in LREE.

REE contents of the Hamchang granite are low (126 $-127 \mathrm{ppm})$ in the low-alkali group of the R22 grano- diorites, but high as 405 to $525 \mathrm{ppm}$ in the high-alkali group of the other granites (Table 3 ). The L/HREE ratio is very high being 17 and 23 throughout the two groups of the granitoids, which is caused by high contents of $\mathrm{Ce}$ and La (Table 3). In the REE pattern (Fig. 6), the R22 granodiorites are depleted in all the components, 
Table 4 (continued).

\begin{tabular}{|c|c|c|c|c|c|c|c|c|c|c|c|c|c|}
\hline \multirow[b]{2}{*}{ Element: } & \multicolumn{9}{|c|}{ Hamchang Granite } & \multicolumn{3}{|c|}{ Gigye Granite } & \multirow{2}{*}{$\begin{array}{l}\begin{array}{l}\text { Hapcheon } \\
\text { syenite }\end{array} \\
\text { K33B } \\
\end{array}$} \\
\hline & K20B & K21B & K23B & $\mathrm{K} 25 \mathrm{C}$ & K26B & K27B & $\mathrm{K} 27 \mathrm{C}$ & K28B & K29B & K30B & $\mathrm{K} 30 \mathrm{C}$ & $\mathrm{K} 32 \mathrm{~B}$ & \\
\hline $\mathrm{SiO}_{2}$ & 64.86 & 64.48 & 63.12 & 63.38 & 61.37 & 58.81 & 63.57 & 66.03 & 64.52 & 73.39 & 74.38 & 72.47 & 58.69 \\
\hline $\mathrm{TiO}_{2}$ & 0.46 & 0.29 & 0.64 & 0.49 & 0.64 & 0.60 & 0.66 & 0.56 & 0.63 & 0.29 & 0.12 & 0.31 & 1.00 \\
\hline $\mathrm{Al}_{2} \mathrm{O}_{3}$ & 16.27 & 18.15 & 18.67 & 18.46 & 18.69 & 19.77 & 17.71 & 16.52 & 16.61 & 13.27 & 12.84 & 13.39 & 19.56 \\
\hline $\mathrm{Fe}_{2} \mathrm{O}_{3}$ & 4.35 & 3.29 & 5.30 & 4.07 & 4.88 & 4.97 & 4.31 & 4.45 & 4.75 & 3.15 & 2.31 & 3.09 & 5.64 \\
\hline $\mathrm{MnO}$ & 0.18 & 0.01 & 0.05 & 0.03 & 0.02 & 0.03 & 0.03 & 0.04 & 0.09 & 0.07 & 0.05 & 0.03 & 0.11 \\
\hline $\mathrm{MgO}$ & 0.75 & 0.27 & 0.74 & 0.47 & 0.57 & 0.61 & 0.58 & 0.97 & 0.71 & 0.31 & 0.12 & 0.26 & 0.48 \\
\hline $\mathrm{CaO}$ & 0.21 & 0.13 & 0.19 & 0.05 & 0.32 & 0.09 & 0.21 & 0.27 & 0.37 & 0.07 & 0.05 & 0.05 & 0.31 \\
\hline $\mathrm{Na}_{2} \mathrm{O}$ & 1.69 & 2.30 & 1.79 & 0.70 & 2.45 & 0.75 & 0.93 & 0.78 & 1.93 & 1.76 & 2.82 & 2.29 & 3.21 \\
\hline $\mathrm{K}_{2} \mathrm{O}$ & 3.99 & 5.87 & 4.53 & 6.58 & 5.75 & 6.19 & 6.28 & 4.39 & 3.97 & 3.72 & 4.32 & 3.87 & 4.96 \\
\hline $\mathrm{P}_{2} \mathrm{O}_{5}$ & 0.04 & 0.03 & 0.03 & 0.03 & 0.04 & 0.04 & 0.05 & 0.04 & 0.04 & 0.01 & $<0.01$ & 0.01 & 0.04 \\
\hline $\mathrm{Cr}_{2} \mathrm{O}_{3}$ & $<0.01$ & $<0.01$ & $<0.01$ & $<0.01$ & $<0.01$ & $<0.01$ & $<0.01$ & $<0.01$ & $<0.01$ & $<0.01$ & $<0.01$ & $<0.01$ & $<0.01$ \\
\hline LOI & 5.83 & 4.12 & 4.98 & 4.51 & 4.78 & 6.81 & 4.91 & 6.23 & 6.02 & 3.74 & 2.54 & 3.68 & 6.36 \\
\hline Total & 98.63 & 98.94 & 100.04 & 98.77 & 99.51 & 98.67 & 99.24 & 100.28 & 99.64 & 99.78 & 99.55 & 99.45 & 100.36 \\
\hline $\mathrm{Rb}$ & 118 & 140 & 134 & 164 & 140 & 145 & 145 & 134 & 145 & 161 & 171 & 157 & 105 \\
\hline $\mathrm{Sr}$ & 95 & 68 & 71 & 66 & 112 & 84 & 98 & 56 & 68 & 17 & 8 & 14 & 80 \\
\hline $\mathrm{Ba}$ & 589 & 349 & 668 & 644 & 730 & 846 & 833 & 528 & 560 & 143 & 86 & 77 & 677 \\
\hline $\mathrm{Zr}$ & 396 & 448 & 606 & 427 & 677 & 504 & 509 & 373 & 465 & 812 & 614 & 293 & 280 \\
\hline $\mathrm{Hf}$ & 10.2 & 12.1 & 15 & 11.4 & 16.5 & 12.1 & 12.6 & 9.8 & 12.2 & 26.7 & 21.8 & 12.3 & 6.7 \\
\hline $\mathrm{Nb}$ & 16.4 & 20 & 24 & 16.7 & 23.6 & 23.6 & 23 & 16.5 & 24.2 & 54.8 & 41.4 & 46.5 & 16.1 \\
\hline $\mathrm{Ta}$ & 1.44 & 1.54 & 1.62 & 1.45 & 1.52 & 1.47 & 1.62 & 1.4 & 1.95 & 4.95 & 4.26 & 4.45 & 1.27 \\
\hline $\mathrm{V}$ & 41 & 17 & 42 & 18 & 23 & 29 & 30 & 52 & 39 & 20 & $<5$ & 25 & 25 \\
\hline $\mathrm{Cr}$ & 30 & $<20$ & 30 & $<20$ & $<20$ & $<20$ & $<20$ & 40 & 30 & $<20$ & $<20$ & 20 & $<20$ \\
\hline Co & 39 & 34 & 28 & 41 & 35 & 31 & 36 & 40 & 42 & 83 & 82 & 87 & 34 \\
\hline $\mathrm{Ni}$ & $<20$ & $<20$ & 3210 & $<20$ & $<20$ & $<20$ & $<20$ & $<20$ & $<20$ & $<20$ & $<20$ & $<20$ & $<20$ \\
\hline $\mathrm{Cu}$ & $<10$ & $<10$ & 20 & $<10$ & $<10$ & $<10$ & $<10$ & $<10$ & $<10$ & $<10$ & $<10$ & $<10$ & $<10$ \\
\hline $\mathrm{Zn}$ & 50 & $<30$ & 70 & 50 & 60 & 60 & 50 & 60 & 80 & 120 & 130 & 70 & 40 \\
\hline $\mathrm{Pb}$ & 27 & 22 & 31 & 30 & 34 & 34 & 27 & 24 & 32 & 39 & 42 & 14 & 8 \\
\hline $\mathrm{Ga}$ & 21 & 26 & 25 & 25 & 26 & 27 & 23 & 22 & 24 & 24 & 26 & 24 & 24 \\
\hline $\mathrm{Ge}$ & 1.2 & 1.1 & 1.6 & 1.2 & 1.3 & 1.5 & 1.3 & 1.3 & 1.6 & 1.8 & 2 & 1.7 & 1.2 \\
\hline As & 6 & $<5$ & 6 & $<5$ & $<5$ & $<5$ & $<5$ & $<5$ & $<5$ & $<5$ & $<5$ & 15 & $<5$ \\
\hline Mo & 3 & $<2$ & $<2$ & $<2$ & $<2$ & $<2$ & $<2$ & $<2$ & $<2$ & $<2$ & $<2$ & 2 & $<2$ \\
\hline $\mathrm{Sn}$ & 3 & 3 & 3 & 2 & 3 & 2 & 2 & 2 & 4 & 9 & 10 & 10 & 1 \\
\hline $\mathrm{Sb}$ & 0.4 & $<0.2$ & 1.5 & 0.5 & 0.7 & 0.7 & 0.4 & 0.5 & 0.7 & 0.5 & 0.7 & 0.5 & 0.4 \\
\hline Cs & 3.7 & 1.7 & 5.3 & 2.5 & 2.6 & 2.4 & 2.6 & 4.9 & 5.6 & 4.4 & 2.9 & 5 & 2 \\
\hline $\mathrm{Tl}$ & 0.84 & 0.68 & 1.06 & 1.15 & 1.09 & 1.08 & 0.98 & 0.95 & 1.17 & 1.08 & 1.25 & 1.1 & 0.5 \\
\hline $\mathrm{Bi}$ & 0.2 & 0.1 & 0.4 & $<0.1$ & $<0.1$ & 0.1 & $<0.1$ & $<0.1$ & $<0.1$ & 0.5 & $<0.1$ & 0.3 & $<0.1$ \\
\hline Th & 23.9 & 41.3 & 31.7 & 20.5 & 17.9 & 16.7 & 14.9 & 27 & 34.2 & 30.5 & 27.8 & 21.7 & 13 \\
\hline $\mathrm{U}$ & 3.37 & 3.7 & 3.57 & 2.46 & 2.4 & 2.84 & 2.77 & 3.22 & 4 & 8.38 & 7.63 & 6.54 & 3.02 \\
\hline $\mathrm{La}$ & 82.7 & 179.0 & 93.7 & 63.9 & 71.8 & 58.0 & 56.5 & 65.6 & 99.7 & 30.7 & 27.5 & 17.3 & 38.0 \\
\hline $\mathrm{Ce}$ & 168.0 & 293.0 & 199.0 & 138.0 & 138.0 & 107.0 & 95.1 & 158.0 & 216.0 & 87.6 & 99.1 & 63.1 & 91.4 \\
\hline $\operatorname{Pr}$ & 16.2 & 34.2 & 19.5 & 13.9 & 16.0 & 13.7 & 11.5 & 12.9 & 20.6 & 8.0 & 7.7 & 4.4 & 9.7 \\
\hline $\mathrm{Nd}$ & 51.4 & 106.0 & 62.1 & 46.7 & 53.8 & 47.9 & 38.7 & 40.3 & 66.9 & 27.9 & 27.2 & 14.6 & 35.1 \\
\hline $\mathrm{Sm}$ & 7.71 & 14.30 & 9.48 & 8.10 & 9.15 & 9.08 & 6.56 & 6.11 & 10.70 & 7.11 & 6.62 & 3.58 & 6.65 \\
\hline $\mathrm{Eu}$ & 1.01 & 1.20 & 1.60 & 1.20 & 1.58 & 1.66 & 1.22 & 0.80 & 1.25 & 0.36 & 0.23 & 0.21 & 1.99 \\
\hline LREE & 327.0 & 627.7 & 385.4 & 271.8 & 290.3 & 237.3 & 209.6 & 283.7 & 415.2 & 161.7 & 168.3 & 103.2 & 182.9 \\
\hline $\mathrm{Gd}$ & 4.50 & 6.89 & 6.01 & 5.16 & 6.77 & 6.62 & 4.75 & 3.44 & 6.76 & 8.88 & 8.47 & 4.57 & 4.75 \\
\hline $\mathrm{Tb}$ & 0.79 & 1.27 & 0.99 & 0.88 & 1.11 & 1.05 & 0.78 & 0.59 & 1.16 & 1.76 & 1.68 & 0.86 & 0.78 \\
\hline Dy & 4.34 & 6.77 & 5.35 & 4.63 & 5.72 & 5.32 & 3.98 & 3.50 & 6.61 & 11.00 & 11.20 & 5.54 & 4.26 \\
\hline Ho & 0.82 & 1.21 & 1.02 & 0.86 & 1.07 & 0.98 & 0.74 & 0.68 & 1.22 & 2.38 & 2.41 & 1.23 & 0.80 \\
\hline Er & 2.55 & 3.73 & 3.22 & 2.48 & 3.18 & 2.96 & 2.27 & 2.12 & 3.71 & 8.57 & 8.67 & 4.51 & 2.51 \\
\hline $\mathrm{Tm}$ & 0.39 & 0.55 & 0.51 & 0.36 & 0.47 & 0.44 & 0.34 & 0.33 & 0.58 & 1.52 & 1.49 & 0.85 & 0.39 \\
\hline $\mathrm{Yb}$ & 2.46 & 3.51 & 3.14 & 2.31 & 2.87 & 2.73 & 2.09 & 2.08 & 3.56 & 10.30 & 10.10 & 5.92 & 2.65 \\
\hline $\mathrm{Lu}$ & 0.35 & 0.49 & 0.48 & 0.33 & 0.40 & 0.40 & 0.30 & 0.30 & 0.51 & 1.56 & 1.49 & 0.90 & 0.43 \\
\hline HREE & 16.19 & 24.42 & 20.71 & 17.00 & 21.59 & 20.50 & 15.25 & 13.04 & 24.11 & 45.97 & 45.51 & 24.37 & 16.56 \\
\hline $\mathrm{Y}$ & 24.8 & 37.8 & 30.6 & 22.6 & 32.6 & 28.9 & 23.3 & 20.7 & 37.7 & 70.6 & 76.6 & 33.4 & 21.9 \\
\hline L/HREE & 8.0 & 10.1 & 7.5 & 6.9 & 5.4 & 4.8 & 5.4 & 8.4 & 6.7 & 1.4 & 1.4 & 1.8 & 4.8 \\
\hline REE & 343.2 & 652.1 & 406.1 & 288.8 & 311.9 & 257.8 & 511.6 & 296.7 & 439.3 & 207.7 & 213.8 & 127.6 & 199.5 \\
\hline REE+Y & 368.0 & 689.9 & 436.7 & 311.4 & 344.5 & 286.7 & 534.9 & 317.4 & 477.0 & 278.3 & 290.4 & 161.0 & 221.4 \\
\hline
\end{tabular}

but the other granitoids with similar $\mathrm{SiO}_{2}$ contents are much higher in the REE components.

The Gigye granite could be a split-off part of the Namsan granite. The Namsan granite consists of hypersolvus alkali-feldspar granite in the northern part and subsolvus biotite granite in the southern part. The hypersolvus granite is enriched in REE, ranging from 139 to 466 ppm (Koh et al., 1996). The Gigye granite of our study contains 200-300 ppm REE, not as high as the hypersolvus granite of the Namsan pluton. The L/ HREE ratios range from 2.5 to 4.1 , implying relatively high amounts of HREE (Table 3 ). The REE pattern 


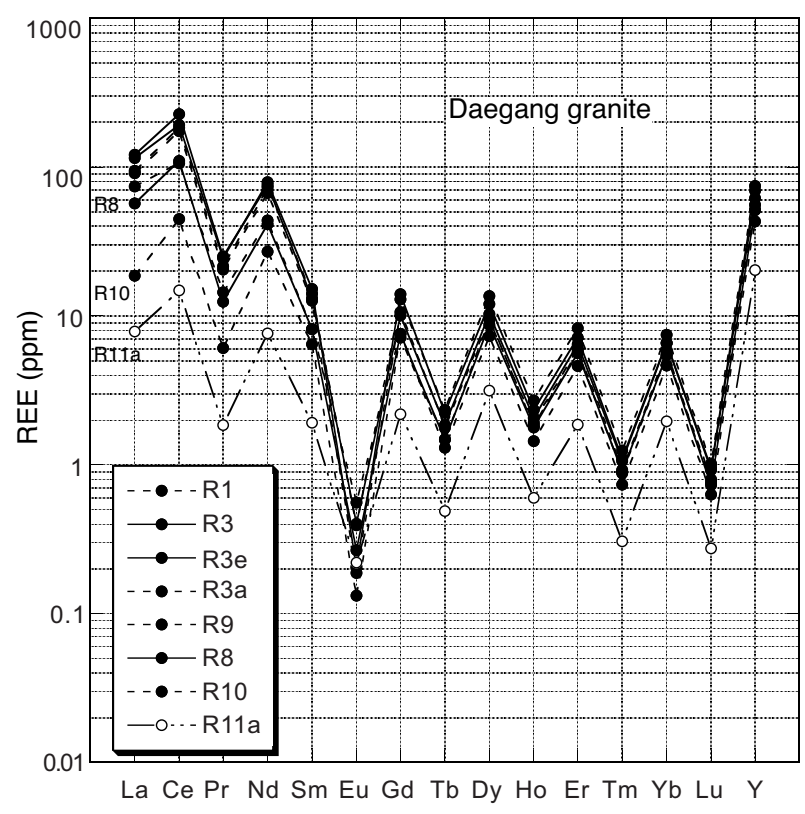

Fig. 5 REE pattern of the Daegang granite.

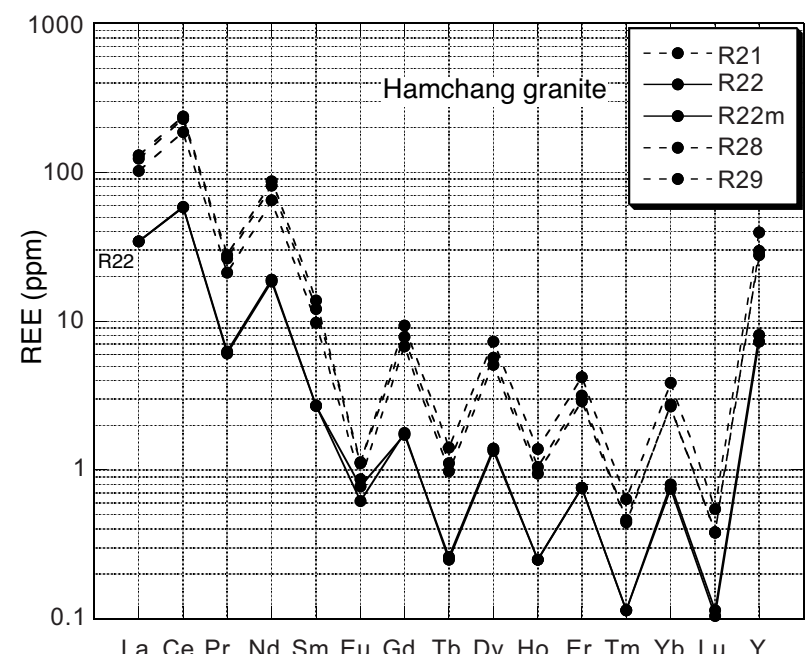

Fig. 6 REE pattern of the Hamchang granite.

shows a strong depletion on the Eu content (Fig. 7).

The Hapcheon syenite is low in the total REE, which varies from 150 to $187 \mathrm{ppm}$. The L/HREE ratio varies from 8 to 15 (Table 3 ). The syenite has weak depletion of Eu, compared with the Gigye granite (Fig. 7).

\section{REE during Weathering}

Annual rain fall of the studied areas varies from 1,200 to 1,600 mm (KMA, 2005), which are similar to that of the southern Jiangxi Province, China, where lateritic REE deposits have been developed in the weathered crust of the Yanshanian granitoids $(\mathrm{Wu}$ et al., 1990). Weathered crust of the studied granitic rocks

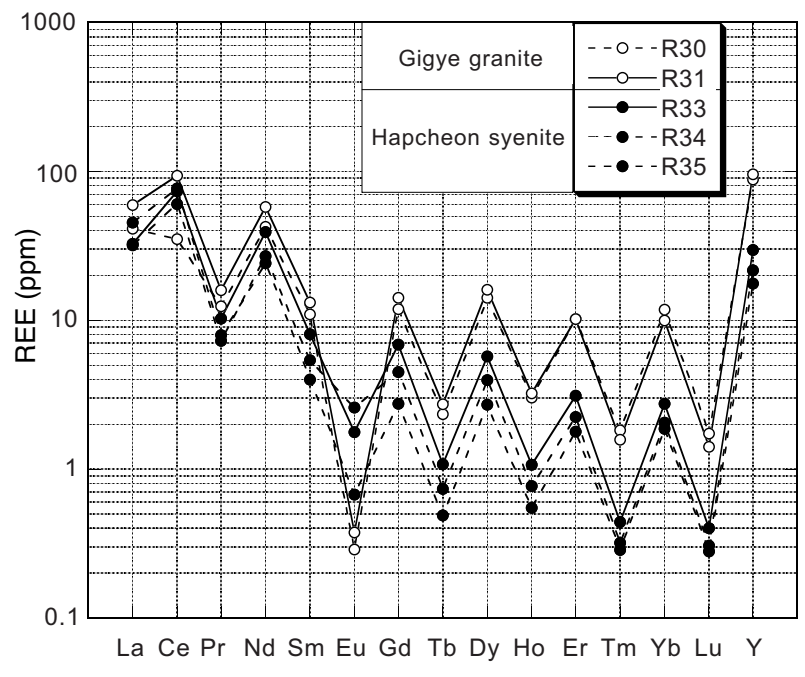

Fig. 7 REE pattern of the Gigye and Hapcheon granites.

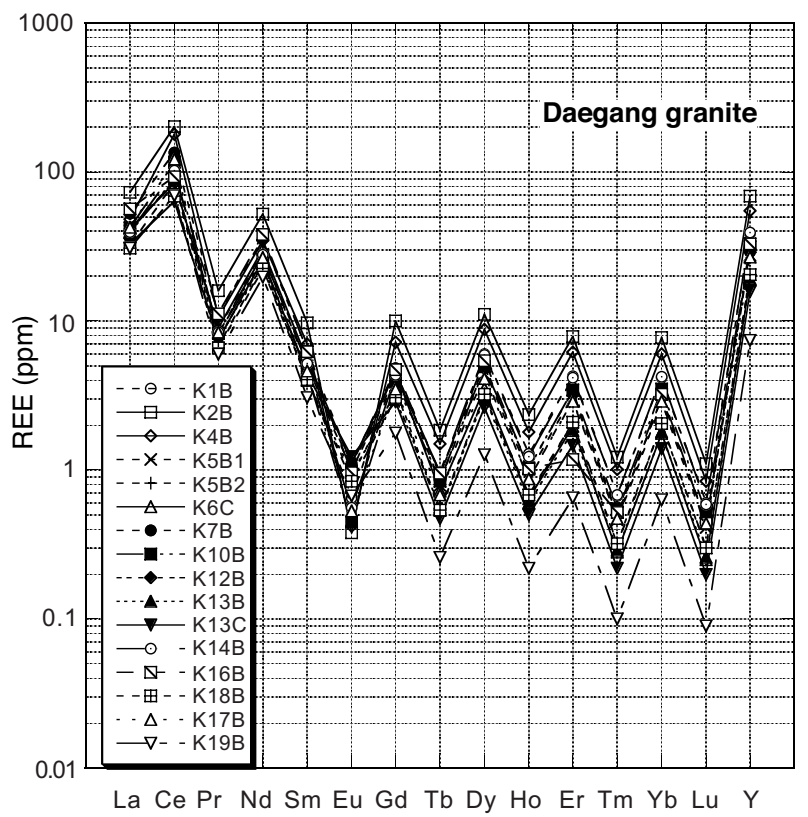

Fig. 8 REE pattern of the soil samples from the Daegang granite.

in South Korea were usually developed down to 2 meters from the surface, which is mostly "B"horizon. The horizons " $A$ " and " $C$ " are not well developed in the studied areas. Besides, angular granitic blocks were observed at many outcrops, suggesting that debris slide modified the crust during the weathering processes.

All the soil samples were chemically analyzed and their mineral assemblages were examined by X-ray powder diffraction method. The samples examined in this study consist mainly of quartz, plagioclase and $\mathrm{K}$ feldspar with small amounts of clay minerals, including kaolinite, halloysite, illite and kaolinite-montmorillonite mixed-layer mineral. Biotite has not been identified although alkalic amphibole is preserved in some 


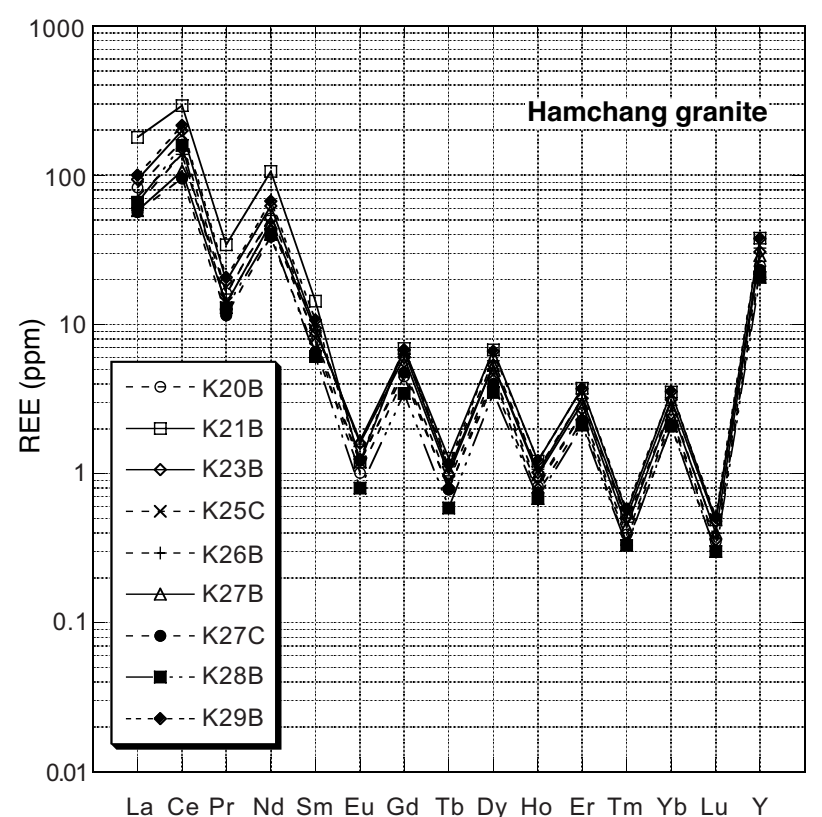

Fig. 9 REE pattern of the soil samples from the Hamchang granite.

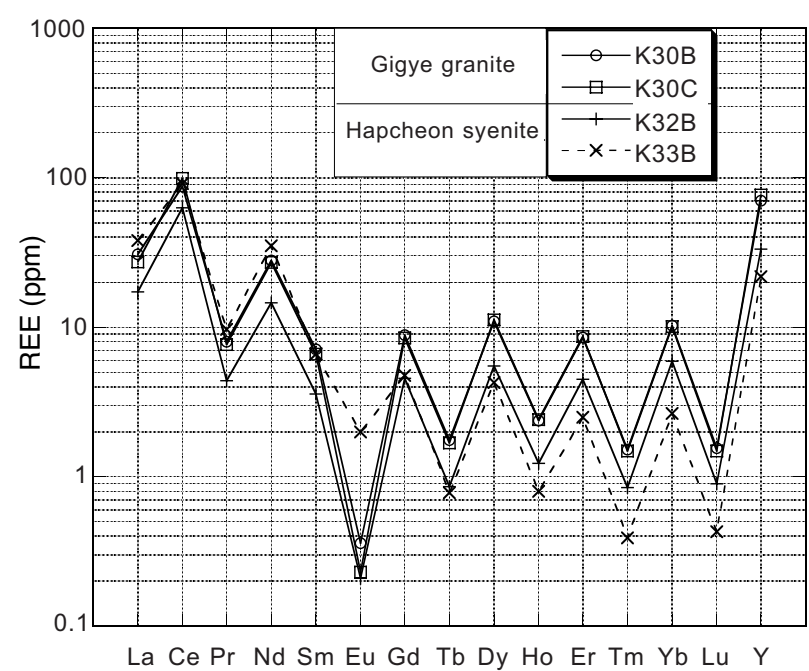

Fig. 10 REE pattern of the soil samples from the Gigye granite and Hapcheon syenite.

samples (e.g., K7B, K30B, K30C, K33B).

Feldspars are abundant in most of the samples, except for relatively clay-rich soils (K5B1, K5B2, K6C, K12B), which are characterized by high LOI (Loss On Ignition) values. $\mathrm{B}$ and $\mathrm{C}$ horizons do not show remarkable difference in their X-ray diffraction patterns. These observations suggest relatively low maturity of weathering in the studied areas. The contents of clay minerals may be approximated by LOI values, which is consistent with X-ray diffraction data. No systematic correlation has been found between the REE contents and the LOI values or assemblages of clay minerals.

Chemical compositions of studied soils are shown in Table 4. In the Daegang body, the REE+Y contents

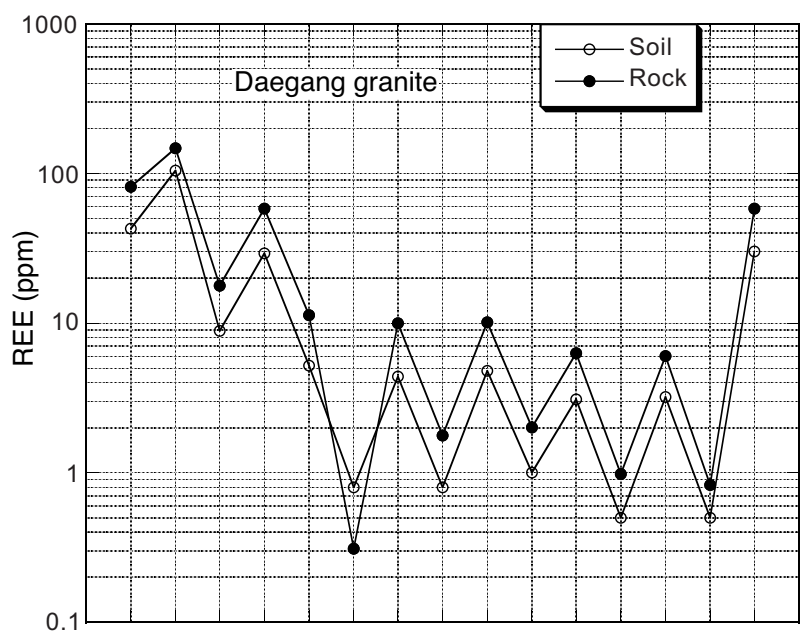

La Ce Pr Nd Sm Eu Gd Tb Dy Ho Er Tm Yb Lu Y

Fig. 11 REE pattern of averaged fresh rocks and soil samples of the Daegang granites.

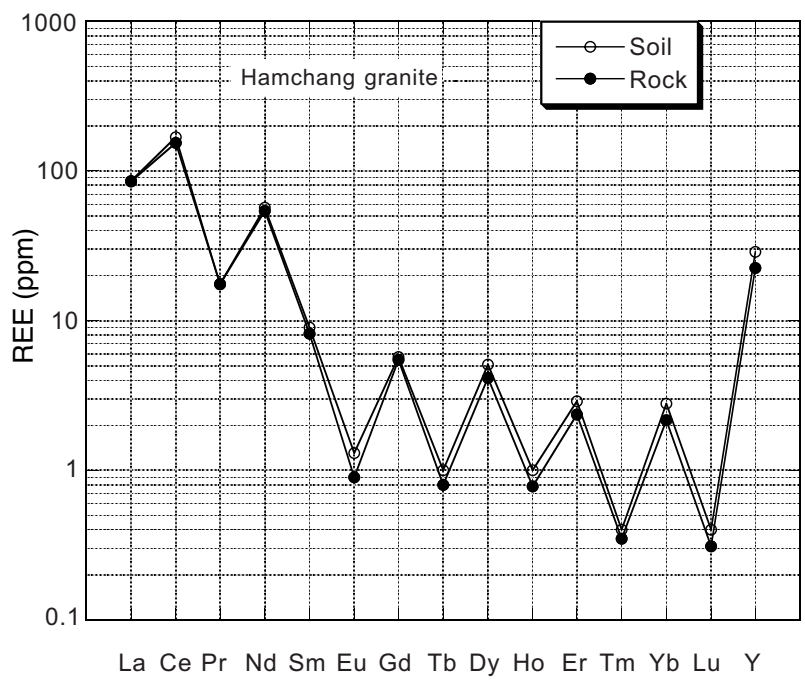

Fig. 12 REE pattern of averaged fresh rocks and soil samples of the Hamchang granites.

are relatively high at two points, $466 \mathrm{ppm}(\mathrm{K} 2 \mathrm{~B})$ and $368 \mathrm{ppm}$ (K4B), but the others are low varying from $290 \mathrm{ppm}$ to $142 \mathrm{ppm}$. L/HREE ratios are generally low, as 10.6 to 2.5 (Table 4). The values are lower than those of the unweathered granites (Fig. 8), which are best shown in Figure 11. REE contents of soil samples are lower than those of the fresh granite samples, except for Eu which is enriched 2-3 times. Similar pattern is obtained on the Gigye granite (Figs. 10, 13), although number of the samples is still small. Here Eu is unchanged, and $\mathrm{Ce}$ is weakly enriched in the soil sample.

Average compositions of the studied rock and soil samples are given in Table 5. The rock/soil ratios of the total REE of the Daegang granite is 1.48 and that 


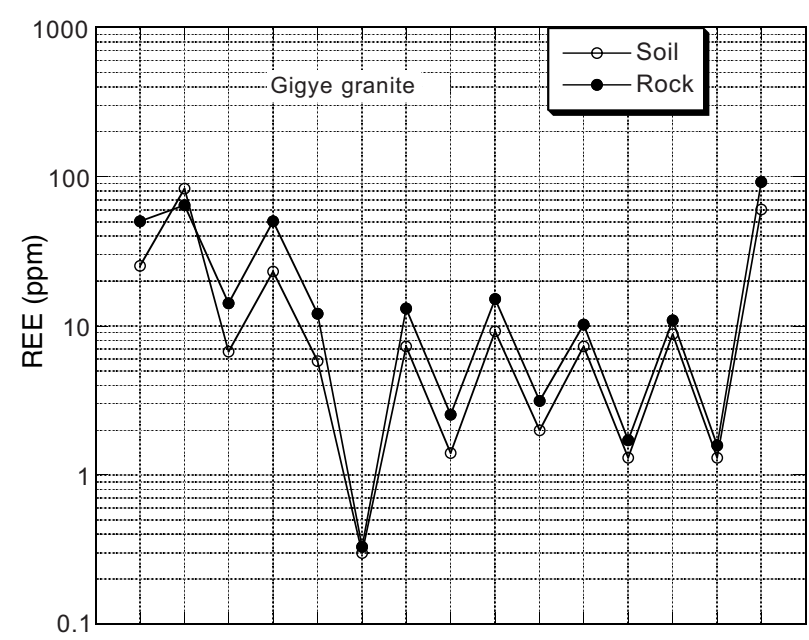

La Ce Pr Nd Sm Eu Gd Tb Dy Ho Er Tm Yb Lu Y

Fig. 13 REE pattern of averaged fresh rock and soil samples of the Gigye granites.

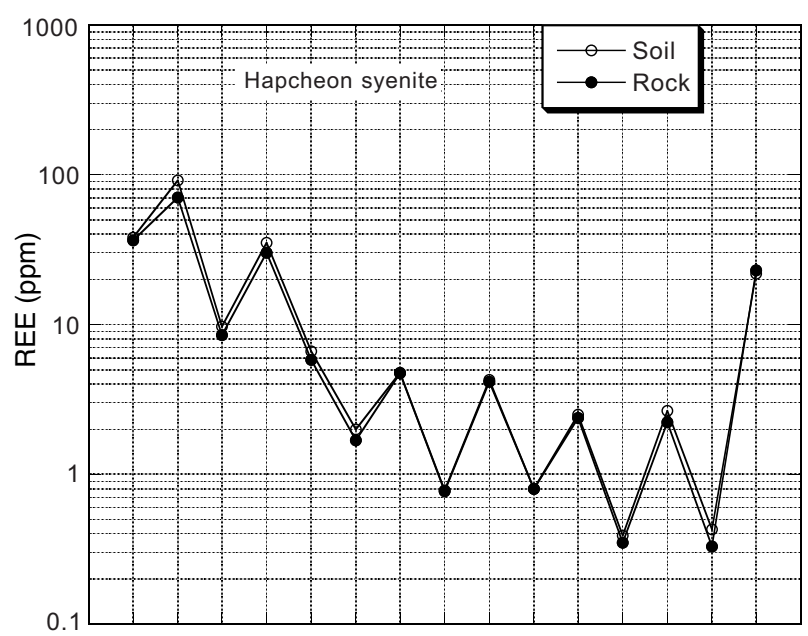

La Ce Pr Nd Sm Eu Gd Tb Dy Ho Er Tm Yb Lu Y

Fig. 14 REE pattern of averaged fresh rock and soil samples of the Hapcheon granites.

Table 5 Averaged REE and Y contents (ppm) of the studied fresh granites and weathered crust.

\begin{tabular}{|c|c|c|c|c|c|c|c|c|}
\hline \multirow{3}{*}{$\begin{array}{l}\text { Pluton } \\
\text { Saml. Nos. } \\
\text { Rock/soil }\end{array}$} & \multirow{2}{*}{\multicolumn{2}{|c|}{$\begin{array}{c}\text { Daegang granite } \\
1-19 \\
\end{array}$}} & \multirow{2}{*}{\multicolumn{2}{|c|}{$\begin{array}{c}\text { Hamchang granite } \\
20-29\end{array}$}} & \multirow{2}{*}{\multicolumn{2}{|c|}{$\begin{array}{r}\text { Gigye granite } \\
30-32 \\
\end{array}$}} & \multirow{2}{*}{\multicolumn{2}{|c|}{$\begin{array}{c}\text { Hapcheon syenite } \\
33-35 \\
\end{array}$}} \\
\hline & & & & & & & & \\
\hline & Rock & Soil & Rock & Soil & Rock & Soil & Rock & Soil \\
\hline $\mathrm{La}$ & 81.7 & 42.8 & 85.0 & 85.7 & 50.3 & 25.2 & 36.6 & 38.0 \\
\hline $\mathrm{Ce}$ & 148.0 & 104.5 & 153.8 & 168.0 & 64.5 & 83.3 & 70.3 & 91.4 \\
\hline $\operatorname{Pr}$ & 17.8 & 8.9 & 17.5 & 17.6 & 14.2 & 6.7 & 8.5 & 9.7 \\
\hline $\mathrm{Nd}$ & 58.2 & 29.3 & 54.2 & 57.1 & 50.1 & 23.2 & 30.1 & 35.1 \\
\hline $\mathrm{Sm}$ & 11.3 & 5.2 & 8.2 & 9.0 & 12.1 & 5.8 & 5.8 & 6.7 \\
\hline $\mathrm{Eu}$ & 0.3 & 0.8 & 0.9 & 1.3 & 0.3 & 0.3 & 1.7 & 2.0 \\
\hline LREE & 317.3 & 191.5 & 319.6 & 338.7 & 191.5 & 144.5 & 153.0 & 182.8 \\
\hline $\mathrm{Gd}$ & 10.0 & 4.4 & 5.5 & 5.7 & 13.1 & 7.3 & 4.7 & 4.8 \\
\hline $\mathrm{Tb}$ & 1.8 & 0.8 & 0.8 & 1.0 & 2.5 & 1.4 & 0.8 & 0.8 \\
\hline Dy & 10.1 & 4.8 & 4.2 & 5.1 & 15.1 & 9.2 & 4.1 & 4.3 \\
\hline Но & 2.0 & 1.0 & 0.8 & 1.0 & 3.1 & 2.0 & 0.8 & 0.8 \\
\hline $\mathrm{Er}$ & 6.3 & 3.1 & 2.4 & 2.9 & 10.2 & 7.3 & 2.4 & 2.5 \\
\hline $\mathrm{Tm}$ & 1.0 & 0.5 & 0.4 & 0.4 & 1.7 & 1.3 & 0.4 & 0.4 \\
\hline $\mathrm{Yb}$ & 6.0 & 3.2 & 2.2 & 2.8 & 10.9 & 8.8 & 2.2 & 2.7 \\
\hline $\mathrm{Lu}$ & 0.8 & 0.5 & 0.3 & 0.4 & 1.6 & 1.3 & 0.3 & 0.4 \\
\hline HREE & 38.0 & 18.3 & 16.4 & 19.3 & 58.3 & 38.6 & 15.7 & 16.6 \\
\hline REE & 355.3 & 209.8 & 336.0 & 358.0 & 249.8 & 183.1 & 168.7 & 199.4 \\
\hline $\mathrm{Y}$ & 58.2 & 30.1 & 22.5 & 28.8 & 92.1 & 60.2 & 23.0 & 21.9 \\
\hline $\mathrm{REE}+\mathrm{Y}$ & 413.5 & 239.9 & 358.5 & 386.8 & 341.9 & 243.3 & 191.7 & 221.3 \\
\hline L/HREE & 8.4 & 10.5 & 19.5 & 17.6 & 3.3 & 3.7 & 9.7 & 11.0 \\
\hline
\end{tabular}

of the Gigye granite is 1.36 . It may be concluded in these two granitic bodies that REE are generally eroded out during the weathering.

On the other two granitic bodies, the loss-and-gain during the weathering is unclear, although all the HREE looks slightly enriched during the weathering in the Hamchang granite (Figs. 9, 12, 14). The rock/soil ratios of the averaged total REE contents (Table 5) are 0.94 in the Hamchang granite and 0.85 in the Hapcheon syenite. Thus, the REEs are most enriched in the 
Hapcheon syenite during weathering in the studied areas.

\section{Concluding Remarks}

Alkaline granites and their weathered crust were examined chemically. The granites are relatively high in trace amounts of REE + Y. The weathered crusts show no evidence of REE-enrichment but often depleted relative to the unweathered host rocks. Although ages of the studied granites are Triassic to Paleogene, the weathered crusts seem to have formed in the Quaternary by uplifting of the Korean Peninsula. This short time-span of the weathering may be the main reason of no accumulation of REE in the studied weathered crust.

\section{Appendix: REE contents of rocks from the former Chungju REE mine}

The world largest REE concentration has been known in the REE-magnetite deposits at Bayan Obo in the Inner Mongolia, China. Similar showings have been known near Chungju in the middle part of South Korea, and they are developed within the Gyemyeongsan Formation of a gneiss-schist complex (Kim et al., 1994). The Chungju iron mine is one of the REE ore deposits in the Ogcheon belt, South Korea.

The REE deposit of the mine mainly contains CeLa, Ta-Nb, Y, Y-Nd and Nd-Th group minerals (Park and Kim, 1995). More than 15 REE-bearing minerals were reported from the mine: they are allanite, fergusonite, thorite, bastnaesite, euxenite, polyclase, monazite, columbite, $\mathrm{Nb}$-rutile, okanogranite, sphene, zircon, illmenite and so on. According to the characteristics of the mineral association, the REE ore deposit can be divided into four ore types: zircon-REE, allanite-REE, feldspar-REE and fluorite-REE types. The Sm-Nd isochron age of the REE deposit was reported to be $330 \mathrm{Ma}$ (Park and Kim, 1995).

This mine is now abandoned and the surface is now used for crushing stone mining for construction purposes. A brief stop was made here to collect samples to estimate bulk composition of REE-bearing outcrop. The 10 hand specimens of Appendix-1 were arbitrary collected, crushed and analyzed for major (XRF) and trace elements (ICP-ICP/MS).

The analyzed samples of more or less a fist size contains more than 6,900 ppm REE+Y (K14E, Appendix-2), which are banded granitic and amphibolitic gneisses. The second highest is light gray gneiss (K14J) of $>5,500 \mathrm{ppm}$. Pink felsic gneiss (K14C) is also high as $>3,700 \mathrm{ppm}$. An average of these analyses, excluding $\mathrm{K} 14 \mathrm{~K}$ because it is a thin vein aplite, is as follows:

LREE > 1,700 ppm, HREE> 354 ppm (LREE/ HREE=4.8), and $\mathrm{Y}>362 \mathrm{ppm}$

These figures may represent an average composition of the ore deposit, and the total REE $+\mathrm{Y}$ content, 2,416 ppm, is one-order of magnitude lower than that of the Bayan-Obo REE-magnetite mine in China (Drew et al., 1990).

\section{References}

Choe, W. H. and Jwa, Y. J. (1998) Petrochemical characteristics of the granites in the Jeomchon area. Jour. Petrol. Soc. Korea, 7, 37-52 (in Korean with English abstract).

Choi, H.I., Lee, B.J., Hwang, S.G., Choi, P., Song, K.Y. and Kim, D.H. (1993) Geological Report of the Nongam Sheet $(1: 25,000)$. Korea Inst. Energy \& Resources, Seoul, 40p (in Korean with English abstract).

Choo, S.H. and Kim, S.J. (1986) Research on Rb/Sr isotope age dating of Youngnam massif (II), Gneiss and gneissic granitoids from the southern part of Mt. Jiri. KIGAM Research Report, KR-86-7, 7-33 (in Korean with English abstract).

Drew, L.J., Meng, Q., and Sun, W. (1990) The Bayan Obo iron-rare earth-niobium deposits, Inner Mongolia, China. Lithos, 26, 43-65.

Huang, D-H., Wu, C-Y., and Han, J-Z. (1989) REE geochemistry and mineralization characteristics of the Zudong and Guanxi granites, Jiangxi Province. Acta Geol. Sinica, 2, 139-157.

Hwang, B.H. (2004) Petrology, isotope and petrogenesis of the granitic rocks in the southern Gyeongsang basin. PhD theis, Pusan National Univeristy, 306p (in Korean with English abstract).

Hwang, S.G., Lee, B.J. and Yoo, B.C. (1992) A phyllonite zone exposed in Sangju area: an ultramylonite caused by reaction softening. Jour. Geol. Soc. Korea, 28, 410425 (in Korean with English abstract).

Ishihara, S. (1977) The magnetite-series and ilmenite-series granitic rocks. Mining Geol., 27, 293-305.

Ishihara, S. and Murakami, H. (2005) An attractive mineral resource: heavy rare-earth - Are the ion-absorption-type deposits enough to support the modern hightechnology industry? Geology News, no. 609, 6-18 (in Japanese).

Ishihara, S., Jin, M.S. and Terashima, S. (2005) Mo-related adakitic granitoids from non-island-arc setting: Jecheon pluton of South Korea. Resource Geology, 55, 385-396.

Jin, M.S., Lee, Y. S. and Ishihara, S. (2001) Granitoids and their magnetic susceptibility in South Korea. Resource Geol., 51, 189-203.

Jwa, Y-J. (2004) Possible source rocks of Mesozoic granites in South Korea: implications for crustal evolution in NE Asia. Trans. Royal Soc. Edinburgh: Earth Sci., 95, 181-198.

Kamitani, M. and Hirano, H. ed. (1994) Study on rareearth resources in weathered granitoids in Thailand. Rept. Intern. Res. Develp. Coop., ITIT Project n. 901-2, Geol. Surv. Japan, 201 p. 
Kim, C.B. and Kim, Y.J. (1990) Geochronology and petrochemistry of foliated granite between Damyang and Jinan. Jour. Korean Inst. Mining Geol., 23, 233-244 (in Korean with English abstract).

Kim, C. B. and Turek, A. (1996) Advances in U-Pb zircon geochronology of Mesozoic plutonism in the southwestern part of Ryeongnam massif, Korea. Geochem. Jour., 30, 323-338.

Kim, C.B., Kim, Y.J. and Hong, S.S. (1990) Geochemical study on foliated granite in the Damyang-Jinan area. Jour. Korean Inst. Mining Geol., 23, 87-104 (in Korean with English abstract).

Kim, G.S., Park, M.E. and Enjoji, M. (1994) Banded and massive iron mineralization in Chungju mine (I): Geology and ore petrography of iron deposit. Econ. Environ. Geol., 27, 523-535.

Kim, Y-J., Cho, D-L. and Lee, C-S. (1998) Petrology, geochemistry and tectonic implication of the A-type Daegang granite in the Namwon area, southwestern part of the Korean peninsula. Econ. Environ. Geol., 31, 399-413 (in Korean with English abstract).

KMA (Korea Meterological Adminstration) (2005) Annual Climatological Report 2004. KMA. 11-1360000000016-10, p. 293.

Koh, J. S., Yun, S. H. and Lee, S.W. (1996) Petrology and geochemical characteristics of A-type granite with particular reference to the Namsan Granite, Kyeonju. Jour. Petrol. Soc. Korea, 5, 142-160 (in Korean with English abstract).

Le Maitre, R.W., Bateman, P., Dudek, A., Keller, J., Lameyre, J., Le Bas, M. J., Sabine, P.A., Schmid, R.,
Sorensen, H., Streckeisen, A., Wooddey, A.R. and Zanettin, B. (1989) A Classification of Igenous Rocks and Glossary of Terms. Blackwell, Oxford, 193 p.

Lee, M.J., Lee, J.I. and Lee, M.S. (1995) Mineralogy and major element geochemistry of A-type alkali granite in the Kyeongju area, Korea. Jour. Geol. Soc. Korea, 31, 583-607.

Park, M.E. and Kim, G.S. (1995) Genesis of the REE ore deposits, Chungju district, Korea: Occurrence features and geochemical characteristics. Econ. Environ. Geol., 28, 599-612 (in Korean with English abstract).

Sagong, H., Kwon, S.-T. and Lee, J.-H. (2005) Mesozoic episodic magmatism in South Korea and its tectonic implication. Tectonics, 24, TC5002, doi:10.1029/ 2004TC001720.

Turek, A. and Kim, C.B. (1995) U-Pb zircon ages of Mesozoic plutons in the Damyang-Geochang area, Ryeongnam massif, Korea. Geochem. Jour., 29, 243258.

Whalen, J. B., Currie, K.L.and Chappell, B.W. (1982) Atype granites: geochemical characteristics, discrimination and petrogenesis. Contrib. Mineral. Petrol., 95, 407-419.

Wu, C-Y., Huang, D-H. and Guo, Z-X. (1990) REE geochemistry in the weathered crust of granites, Longnan area, Jiangxi Province. Acta Geol. Sinica, 3, 193-210.

Received June 26, 2006

Accepted September 21, 2006 


\section{韓国における幾つかのアルカリ花崗岩類とその風化殼のレアアース資源評価 石原舜三, 佐藤興平, 左 容周, 金 鍾善 \\ 要 旨}

花崗岩風化殼へのREEの濃集を見るために，韓国の中部の花崗岩地域 4 箇所 (平均降雨量 $1,300 \mathrm{~mm} /$ 年) で予察 的な調査を実施した。嶺南帯と沃川帯境界部に沿って伸長する三畳紀の片麻状黒雲母花崗岩（帶江岩体）はREEに 富み, 平均 $414 \mathrm{ppm} \mathrm{REE}+\mathrm{Y}, \mathrm{LREE} / \mathrm{HREE}=4.8$ ある。沃川帯の三畳紀咸昌（ハムチャン）花崗岩類は，アルカリ と REEに富むグループ (平均 $359 \mathrm{ppm} \mathrm{REE}+\mathrm{Y}, \mathrm{L} / \mathrm{HREE}=19.0$ ) と, 低いグループ (平均 $127 \mathrm{ppm} \mathrm{REE}+\mathrm{Y}, \mathrm{L} / \mathrm{HREE}$ =23）に分けられる。他方, 嶺南帯南東部の初期ジュラ紀の陝川 (ハプチョン) 閃長岩はREE+Y $(171 \sim 217 \mathrm{ppm})$ に 乏しい。慶尚盆地の杞溪（キゲ）花崗岩は南山アルカリ花崗岩の断層による片割れと言われているが，その含有量 は200〜300 ppm REE+Yであるに過ぎない.

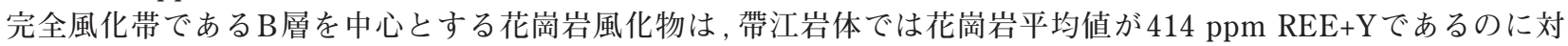
し, 風化土堙は $240 \mathrm{ppm} \mathrm{REE}+\mathrm{Y}$ で希土類元素が減少しており，風化課程における REEの溶脱が考えられる。杞溪 岩体でも $342 \mathrm{ppm} ら 243 \mathrm{ppm} \mathrm{REE}+\mathrm{Y}$ 希土類元素は減少している。その他の岩体での増減は不鮮明である。韓国 では花崗岩の風化課程で希土類元素の明瞭な濃集は見られない.

忠州鉄鉱床の採掘跡におけるランダム サンプリングによると,この鉱床は平均 $0.2 \% \mathrm{REE}+\mathrm{Y}$ 程度の鉱石を保有し ていたと推定される.

\section{付録:漢字対照表}

Daegang (帶江) granite, Gokseong-gun (谷城郡), Jeollanam-do (全羅南道), Hamchang (咸昌) granite, Sangju (尚州), Gyeongsangbuk-do(慶尚北道), Gigye(杞溪) granite, Pohang(浦項), Hapcheon (陝川) syenite, Gyeosangnam-do（慶尚南道）

Appendix Table 1 Rock description and magnetic susceptibility (MS) of the analyzed samples from the former Chungju mine surface.

\begin{tabular}{l}
\hline \hline Numbers $\quad$ Rock description and magnetic susceptibility(MS) \\
\hline K14A: $10 \times 12 \mathrm{~cm}$, magnetite/ green banded gneiss. MS $>894 \times 10^{-3} \mathrm{SI}$ \\
K14B: $7 \times 12 \mathrm{~cm}$, magnetite-amphibolite gneiss. MS $=156 \times 10^{-3} \mathrm{SI}$ \\
K14C: $6 \times 7 \mathrm{~cm}$, very fine, pink felsic gneiss. MS $>53.9 \times 10^{-3} \mathrm{SI}$ \\
K14E: $8 \times 10 \mathrm{~cm}$, pink granitic vein/amphibolite $(3 / 2)$ \\
K14F: $7 \times 9 \mathrm{~cm}$, mudium, epidote-chlorite granodioritic gneiss. MS $=1.5 \times 10^{-3} \mathrm{SI}$ \\
K14G: $9 \times 16 \mathrm{~cm}$ pink biotite gneiss xenolith in pink granite. MS $>15.1 \times 10^{-3} \mathrm{SI}$ \\
K14H: $6 \times 12 \mathrm{~cm}$, pink banded gneiss. MS $>46.6 \times 10^{-3} \mathrm{SI}$ \\
K14 I $5 \times 10 \mathrm{~cm}$, quartz-calcite-rich gneissic band. \\
K14 J: $10 \times 10 \mathrm{~cm}$, light gray skarn-gneiss. MS $>70.2 \times 10^{-3} \mathrm{SI}$ \\
K14K: $5 \mathrm{~cm}$ wide veinlet of pink fine-grained aplitic granite. MS $>5.6 \times 10^{-3} \mathrm{SI}$. \\
\hline
\end{tabular}

MS measured by Kappameter, KT-5. 
Appendix Table 2 Major (XRF) and trace (ICP-ICP/MS) element compositions of rocks and ores from the Chungju mine.

\begin{tabular}{|c|c|c|c|c|c|c|c|c|c|c|}
\hline Element: & K14A & K14B & K14C & K14E & K14F & K14G & $\overline{\mathrm{K} 14 \mathrm{H}}$ & K14I & K14J & K14K \\
\hline $\mathrm{SiO}_{2}$ & 26.48 & 45.97 & 73.39 & 60.33 & 66.09 & 74.39 & 68.92 & 77.42 & 70.10 & 75.37 \\
\hline $\mathrm{TiO}_{2}$ & 0.27 & 3.46 & 0.40 & 1.51 & 0.27 & 0.12 & 0.25 & 0.21 & 0.41 & 0.02 \\
\hline $\mathrm{Al}_{2} \mathrm{O}_{3}$ & 1.07 & 14.41 & 8.81 & 14.34 & 16.22 & 12.40 & 9.38 & 2.34 & 8.20 & 13.29 \\
\hline $\mathrm{Fe}_{2} \mathrm{O}_{3}$ & 68.07 & 15.18 & 6.67 & 8.08 & 4.02 & 1.89 & 6.76 & 8.23 & 8.48 & 0.57 \\
\hline $\mathrm{MnO}$ & 0.27 & 0.13 & 0.04 & 0.18 & 0.08 & 0.04 & 0.15 & 0.11 & 0.16 & 0.01 \\
\hline $\mathrm{MgO}$ & 0.62 & 5.14 & 0.21 & 1.55 & 0.47 & 0.29 & 1.04 & 0.31 & 1.17 & 0.11 \\
\hline $\mathrm{CaO}$ & 3.11 & 4.84 & 1.96 & 2.95 & 5.30 & 0.99 & 4.24 & 6.83 & 4.24 & 1.12 \\
\hline $\mathrm{Na}_{2} \mathrm{O}$ & $<0.01$ & 5.28 & 1.15 & 2.43 & 3.65 & 2.40 & 0.58 & 0.09 & 2.64 & 3.82 \\
\hline $\mathrm{K}_{2} \mathrm{O}$ & 0.02 & 2.73 & 5.52 & 7.03 & 2.18 & 6.14 & 5.98 & 1.15 & 2.78 & 5.01 \\
\hline $\mathrm{P}_{2} \mathrm{O}_{5}$ & 0.04 & 2.00 & $<0.01$ & 0.33 & 0.06 & 0.01 & $<0.01$ & $<0.01$ & $<0.01$ & 0.01 \\
\hline $\mathrm{Cr}_{2} \mathrm{O}_{3}$ & $<0.01$ & $<0.01$ & $<0.01$ & 0.01 & $<0.01$ & $<0.01$ & $<0.01$ & $<0.01$ & $<0.01$ & $<0.01$ \\
\hline LOI & 0.19 & 0.64 & 1.03 & 1.02 & 1.31 & 0.91 & 1.34 & 2.05 & 0.78 & 0.65 \\
\hline Total & 100.14 & 99.78 & 99.18 & 99.76 & 99.65 & 99.58 & 98.64 & 98.74 & 98.96 & 99.98 \\
\hline $\mathrm{Rb}$ & 4 & 188 & 161 & 181 & 83 & 148 & 151 & 37 & 132 & 162 \\
\hline $\mathrm{Sr}$ & 28 & 214 & 34 & 152 & 356 & 171 & 57 & 35 & 39 & 143 \\
\hline $\mathrm{Ba}$ & 6 & 198 & 165 & 829 & 319 & 890 & 240 & 35 & 86 & 727 \\
\hline $\mathrm{Zr}$ & 953 & 301 & $>10000$ & 829 & 596 & 1060 & 2800 & 2620 & $>10000$ & 89 \\
\hline Hf & 25.6 & 7.4 & 311 & 23.2 & 15.8 & 27.9 & 75 & 70.3 & 291 & 2.7 \\
\hline $\mathrm{Nb}$ & 92.7 & 60.8 & $>1000$ & 280 & 62.8 & 171 & 449 & 513 & $>1000$ & 32.7 \\
\hline $\mathrm{Ta}$ & 7.31 & 4.07 & 143 & 19.4 & 3.96 & 15.9 & 32.4 & 33 & 120 & 6.01 \\
\hline $\mathrm{V}$ & 11 & 142 & $<5$ & 76 & 6 & $<5$ & $<5$ & $<5$ & $<5$ & $<5$ \\
\hline $\mathrm{Co}$ & 80 & 50 & 118 & 95 & 152 & 211 & 136 & 232 & 150 & 249 \\
\hline $\mathrm{Cu}$ & $<10$ & $<10$ & $<10$ & $<10$ & $<10$ & 20 & $<10$ & $<10$ & $<10$ & $<10$ \\
\hline $\mathrm{Zn}$ & 180 & 70 & $<30$ & 60 & $<30$ & $<30$ & 30 & $<30$ & $<30$ & $<30$ \\
\hline $\mathrm{Pb}$ & $<5$ & $<5$ & 10 & 14 & 7 & 11 & 7 & $<5$ & 7 & 7 \\
\hline $\mathrm{Ga}$ & 16 & 24 & 33 & 45 & 27 & 20 & 34 & 17 & 44 & 14 \\
\hline $\mathrm{Ge}$ & 18.4 & 2 & 4 & 26.3 & 1.8 & 1.7 & 5.1 & 3.9 & 5.6 & 0.8 \\
\hline As & 10 & 19 & 9 & 143 & $<5$ & $<5$ & $<5$ & 6 & 18 & $<5$ \\
\hline Mo & $<2$ & 3 & $<2$ & 2 & 20 & 32 & $<2$ & $<2$ & $<2$ & $<2$ \\
\hline $\mathrm{Sn}$ & 17 & 2 & 125 & 13 & 11 & 6 & 24 & 77 & 123 & 8 \\
\hline $\mathrm{Sb}$ & 3.4 & 1 & 0.9 & 5.4 & 0.5 & 1.6 & 0.8 & 0.8 & 1.4 & 0.3 \\
\hline $\mathrm{Cs}$ & 0.7 & 4 & 0.3 & 4.1 & 0.9 & 1.1 & 0.4 & $<0.1$ & 0.4 & 0.5 \\
\hline $\mathrm{Tl}$ & 0.06 & 0.42 & 0.25 & 0.61 & 0.39 & 0.43 & 0.36 & 0.05 & 0.1 & 0.37 \\
\hline $\mathrm{Bi}$ & 0.2 & $<0.1$ & 0.2 & 0.3 & 0.1 & $<0.1$ & $<0.1$ & $<0.1$ & 0.2 & $<0.1$ \\
\hline $\mathrm{Th}$ & 7.33 & 5.21 & 257 & 75.7 & 37.7 & 31.3 & 85.5 & 59.8 & 242 & 14 \\
\hline U & 4.92 & 2.92 & 71.7 & 4.38 & 2.36 & 5.28 & 33.9 & 20.4 & 65.4 & 0.98 \\
\hline $\mathrm{La}$ & 33.1 & 71.3 & 415.0 & 1760.0 & 139.0 & 122.0 & 130.0 & 164.0 & 865.0 & 38.3 \\
\hline $\mathrm{Ce}$ & 67.8 & 156.0 & 853.0 & $>3000$ & 242.0 & 233.0 & 313.0 & 354.0 & 1830.0 & 65.1 \\
\hline $\operatorname{Pr}$ & 6.7 & 20.8 & 115.0 & 355.0 & 25.6 & 26.4 & 35.3 & 54.7 & 218.0 & 6.8 \\
\hline $\mathrm{Nd}$ & 22.2 & 84.4 & 517.0 & 1190.0 & 81.9 & 88.6 & 139.0 & 234.0 & 761.0 & 21.6 \\
\hline $\mathrm{Sm}$ & 4.6 & 17.1 & 141.0 & 174.0 & 12.7 & 19.2 & 35.9 & 60.9 & 163.0 & 3.8 \\
\hline $\mathrm{Eu}$ & 0.5 & 6.3 & 7.6 & 15.3 & 2.3 & 1.4 & 1.7 & 3.1 & 7.7 & 0.7 \\
\hline LREE & 134.9 & 355.9 & 2048.6 & 6494.3 & 503.5 & 490.6 & 654.9 & 870.7 & 3844.7 & 136.4 \\
\hline $\mathrm{Gd}$ & 4.29 & 14.50 & 150.00 & 106.00 & 7.84 & 18.70 & 42.90 & 65.60 & 168.00 & 2.76 \\
\hline $\mathrm{Tb}$ & 0.74 & 2.19 & 31.50 & 10.20 & 1.07 & 3.42 & 9.58 & 12.70 & 30.60 & 0.43 \\
\hline Dy & 4.26 & 10.50 & 195.00 & 42.90 & 4.60 & 20.00 & 63.20 & 76.30 & 176.00 & 2.10 \\
\hline Ho & 0.85 & 1.80 & 38.80 & 7.00 & 0.76 & 3.79 & 13.70 & 15.80 & 34.80 & 0.38 \\
\hline $\mathrm{Er}$ & 2.68 & 4.76 & 121.00 & 19.50 & 2.10 & 11.20 & 44.00 & 49.50 & 108.00 & 1.11 \\
\hline $\mathrm{Tm}$ & 0.46 & 0.64 & 18.60 & 2.60 & 0.29 & 1.66 & 6.59 & 7.56 & 16.80 & 0.16 \\
\hline $\mathrm{Yb}$ & 3.34 & 3.72 & 111.00 & 14.90 & 1.83 & 9.89 & 40.10 & 45.90 & 102.00 & 0.99 \\
\hline $\mathrm{Lu}$ & 0.57 & 0.52 & 14.60 & 1.86 & 0.32 & 1.32 & 5.48 & 6.13 & 13.50 & 0.14 \\
\hline HREE & 17.19 & 38.63 & $>1680.5$ & 204.96 & 18.80 & 69.98 & 225.55 & 279.49 & 649.70 & 8.07 \\
\hline $\mathrm{Y}$ & 26.7 & 51.6 & $>1000$ & 202.0 & 21.1 & 112.0 & 377.0 & 464.0 & $>1000$ & 12.3 \\
\hline HREE+Y & 43.9 & 90.2 & $>1680.5$ & 407.0 & 39.9 & 182.0 & 602.6 & 743.5 & $>1649.7$ & 20.4 \\
\hline $\begin{array}{l}\text { L/HREE } \\
\end{array}$ & 3.1 & 4.0 & $<1.2$ & $>16.0$ & 12.6 & 2.7 & 1.1 & 1.2 & $<2.3$ & 6.7 \\
\hline $\mathrm{REE}+\mathrm{Y}$ & 178.8 & 446.1 & $>3729.1$ & $>6901.3$ & 543.4 & 672.6 & 1257.5 & 1614.2 & $>5494.4$ & 156.8 \\
\hline
\end{tabular}

Analyist: Actlabs, Ltd. Cr and Ni, below 20 ppm 